\title{
Dscam homophilic specificity is generated by high order cis-multimers coupled with trans self-binding of variable Ig1 in Chelicerata
}

Fengyan Zhou ${ }^{1}$, Guozheng Cao ${ }^{1}$, Songjun $\mathrm{Dai}^{1}$, Guo $\mathrm{Li}^{1}$, Hao $\mathrm{Li}^{1}$, Zhu Ding ${ }^{1}$, Shouqing Hou ${ }^{1}$, Bingbing $\mathrm{Xu}^{1}$, Wendong You ${ }^{2}$, Feng Shi ${ }^{1}$, Xiaofeng Yang ${ }^{2}$, Yongfeng $\mathrm{Jin}^{1 *}$

${ }^{1}$ MOE Laboratory of Biosystems Homeostasis \& Protection and Innovation Center for Cell Signaling Network, College of Life Sciences, ${ }^{2}$ Department of Neurosurgery, First Affiliated Hospital, School of Medicine, Zhejiang University, Hangzhou, Zhejiang, ZJ310058, China

* Correspondence should be addressed to Yongfeng Jin: 0086-571-88206479(Tel); 0086-57188206478(Fax); jinyf@zju.edu.cn (e-mail).

Article Type: Research Article

Running Title: Chelicerata Dscams mediate homophilic recognition

Keywords: Down syndrome cell adhesion molecule; isoform diversity; alternative promoter; homophilic binding; multimer; combinatorial specificity; cell identity; self-recognition; selfavoidance; self-non-self discrimination; Chelicerata 


\begin{abstract}
By alternative splicing, Drosophila Down syndrome cell adhesion molecule (Dscam1) encodes tens of thousands of proteins required for establishing neural circuits, while Chelicerata encodes a family of $\sim 100$ shortened Dscam (sDscam) isoforms via alternative promoters. We report that Dscam isoforms interact promiscuously in cis to generate a vast repertoire of combinatorial homophilic recognition specificities in Chelicerata. Specifically, sDscams formed high order cis-multimers without isoform specificity involving the membrane-proximal fibronectin type III (FNIII) 1-3 and transmembrane (TM) domains and associated specifically in trans via antiparallel self-binding of the first variable immunoglobulin (Ig1) domain. We propose that such sDscam combinatorial homophilic specificity is sufficient to provide each neuron with a unique identity for self-non-self discrimination. In many respects, our results amazingly mirror those reported for the structurally unrelated vertebrate protocadherins (Pcdh) rather than for the closely related fly Dscam1. Thus, our findings blur the distinction between the neuronal selfavoidance of invertebrates and vertebrates and provide insight into the basic principles and evolution of metazoan self-avoidance and self-non-self discrimination.
\end{abstract}




\section{Introduction}

Neuronal self-avoidance refers to the tendency of neurites from the same neuron to avoid each other, which is conserved in vertebrates and invertebrates and plays a vital role in neural circuit assembly ${ }^{1,2}$. Also, this process is mediated by strictly homophilic interactions between cell recognition proteins that trigger a neurite-repulsive rather than -adhesive signal. In turn, neuronal self-avoidance requires a molecular mechanism by which each cell discriminates self from non-self ${ }^{1,2}$. Thus, one challenge in deciphering the basis of self-avoidance is elucidating how neurons discriminate self from non-self. One solution to this challenge is to endow each neuron with a unique identity by expressing diverse families of cell adhesion molecules ${ }^{1,2,3}$.

In Drosophila, neuronal self-avoidance is mediated by extraordinary cell recognition molecules of the immunoglobulin superfamily, which are encoded by the Down syndrome cell adhesion molecule (Dscam1) locus via alternative splicing ${ }^{1,3}$. This vast repertoire of Dscam1 recognition molecules is sufficient to bestow a unique molecular identity on each neuron and homophilic interaction specificity, thereby allowing neuronal processes to distinguish between self and non-self ${ }^{4,5}$. If neurites of the same neuron approach each other, neurites with identical Dscam1 isoforms exhibit homophilic interactions, resulting in self-avoidance due to contactdependent repulsion. By contrast, neurites from different neurons express distinct Dscam1 protein repertoires that do not engage in homophilic binding and thus contact each other ${ }^{1,6}$.

Stochastic alternative splicing of Drosophila Dscam1 enables it to encode up to 38,016 distinct isoforms, each of which comprises 1 of 19,008 distinct ectodomains linked to one of two alternative transmembrane regions ${ }^{7,8,9,10}$. Individual neurons stochastically express a 
unique set of distinct Dscam1 isoforms, which may engage in highly isoform-specific homophilic binding between neurons, thus endowing each neuron with a unique molecular identity ${ }^{4,5}$. Dscam isoform diversity plays essential roles in self-recognition and self-avoidance 11, 12, 13. In contrast to insect Dscam1, vertebrate Dscam genes do not produce extensive protein isoforms, and functional studies revealed that mouse Dscam genes are not essential for neuronal self-avoidance ${ }^{14,15}$.

However, another set of genes, the clustered $P c d h$, perform a similar function in vertebrates, and generate enormous cell-surface structural diversity ${ }^{1,2,3,16}$. Pcdhs belong to the cadherin superfamily, the largest and best-established family of cell-adhesion molecules. In human and mouse, 53 and 58 Pcdh proteins are encoded by three tandemly arranged gene clusters of $P c d h \alpha, P c d h \beta$, and $P c d h \gamma$, respectively ${ }^{17,18}$. In contrast to fly Dscam1, the individual variable exon is preceded by an alternative promoter, and differential expression of Pcdh isoforms is achieved by a combination of stochastic promoter selection and alternative splicing 19, 20,21. Like fly Dscam1 isoforms, almost all clustered Pcdh proteins engage in isoform-specific trans homophilic interactions ${ }^{22,23}$. However, in contrast to fly Dscam1 isoforms, which act as a monomer, Pcdh proteins act as multimeric recognition units to expand the adhesive interface $22,23,24,25,26,27,28,29$, which provides a reasonable explanation for the different isoform number between vertebrate Pcdh and fly Dscam1. Deletion of the Pcdh $\gamma$-subcluster or all three clusters caused self-avoidance defects of dendrites and axons ${ }^{30,31}$, positing that Drosophila Dscam1 isoforms and vertebrate clustered Pcdhs employ similar strategies for self-avoidance. Also, vertebrate and Drosophila neurons have solved the self-avoidance problem based on a fundamentally similar principle albeit with a different set of recognition molecules. 
Of particular relevance to the remarkable functional convergence of Drosophila Dscam1 and vertebrate clustered Pcdhs is our recent discovery of a "hybrid" gene family in the subphylum Chelicerata. This gene family was composed of shortened Dscam genes with tandemly arrayed 5 ' cassettes, which encoded $\sim 50-100$ isoforms varying across various Chelicerata species via alternative promoter ${ }^{32,33}$. Since these Chelicerata Dscams lack the Nterminal Ig1-6, 10 domains and FNIII3-4, 6 domains present in classical DSCAM, we refer to this type of Dscam as shortened Dscam (sDscam) to distinguish it from classical Dscam. Based on their different variable 5' cassettes encoding one or two Ig domains, these sDscams can be subdivided into the sDscam $\alpha$ and $\mathrm{sDscam} \beta$ subfamilies. Thus, all sDscam isoforms share the same domain structure but contain variable amino acid sequences within the N-terminal and two Ig domains in the extracellular region. Interestingly, the 5' variable region of Chelicerata sDscams shows remarkable organizational resemblance to that of vertebrate-clustered Pcdhs ${ }^{14}$, 32, 33 . Similar to Drosophila Dscam1 and vertebrate Pcdhs, Chelicerata sDscam are abundantly expressed in the nervous system ${ }^{32,33}$. Because Chelicerata sDscams are remarkably similar to Drosophila Dscam1, and exhibit a remarkable organizational resemblance to the vertebrateclustered Pcdhs, with the latter two proteins both capable of mediating self-recognition and selfavoidance, we speculate that these sDscam isoforms play analogous roles in Chelicerata species. Therefore, a systemic examination of the homophilic recognition specificities of these clustered sDscam isoforms was performed to address their roles in specifying single-cell identity and neural circuit assembly.

In this study, we show that most clustered sDscams can engage in highly specific homophilic interactions via antiparallel self-binding of the variable $\operatorname{Ig} 1$ domain. Moreover, we 
provide compelling evidence that sDscam isoforms can associate promiscuously as high order cis-multimers, which is mediated by the constant FNIII1-3 and transmembrane domains. Based on a large body of experimental evidence and structural modeling, we concluded that sDscams mediate self-recognition via promiscuous cis interactions coupled with strictly homophilic $\operatorname{Ig} 1 / \operatorname{Ig} 1$ interactions in trans, possibly forming an interconnected latticed protein assembly between apposed cell surfaces. We propose that these sDscam homophilic specificities are sufficient to provide the unique single-cell identity necessary for neuronal self-non-self discrimination. Interestingly, in many respects, Chelicerata sDscams show more remarkable parallels with the genetically unrelated vertebrate Pcdhs than to the closely related fly Dscam1. Thus, our findings provide mechanistic and evolutionary insight into self-non-self discrimination in metazoans and enhance our understanding of the general biological principles required for endowing cells with distinct molecular identities.

\section{Results}

\section{Cluster-wide analysis of sDscam-mediated homophilic interactions}

The Mesobuthus martensii sDscam gene clusters encode diverse cell-adhesion proteins: 40 alternate sDscam $\alpha$ isoforms and $55 \mathrm{sDscam} \beta 1, \beta 2, \beta 3, \beta 4, \beta 5$, and $\beta 6$ isoforms (Fig. 1a) ${ }^{32,33}$. To investigate whether sDscam isoforms mediate homophilic binding, we expressed the sDscam proteins in Sf9 cells using an insect baculovirus expression system (Fig. 1b). This system is a powerful tool for investigating homophilic interactions between cell surface adhesion molecules ${ }^{34}$. We prepared $\mathrm{Sf} 9$ cells expressing sDscam, as well as Sf9 cells infected with the parental virus as a negative control and expressing fly Dscam1 as a positive control. 
Sf9 cells expressing constructs encoding full-length sDscam $\beta 6 \mathrm{v} 2$ ( $\beta 6 \mathrm{v} 2 \mathrm{FL}-\mathrm{mCherry})$ or sDscam $\beta 6 \mathrm{v} 2$ lacking the cytoplasmic domain ( $\beta 6 \mathrm{v} 2 \Delta$ cyto-mCherry) exhibited strong cell aggregation (Supplementary Fig. 1a), indicating that the homophilic interaction was mediated by sDscam $\beta 6 \mathrm{v} 2$ in trans independently of the cytoplasmic region. Because our results indicated that deletion of the cytoplasmic tail of sDscam did not significantly affect the formation of cell aggregates (Supplementary Fig. 1a), we used $\Delta$ cyto constructs for all sDscam proteins in the cell aggregation assay.

Except for a few sDscam cDNAs that failed to be cloned possibly due to low expression, we performed a systematic analysis of the homophilic interactions of 85 of the 95 sDscam proteins (sDscam $\alpha, \mathrm{sDscam} \beta 1-\beta 6)$. We found that the feature or size of the cell aggregates varied markedly across sDscam subfamilies (Fig. 1c, d, Supplementary Fig. 1b). Cells expressing each alternate sDscam $\alpha$ showed extensive aggregation for all isoforms tested (Fig. 1d, lane 1-3). By contrast, cells expressing most of the alternate sDscam $\beta 1-\beta 4$ isoforms failed to form aggregates, except for a few isoforms (Fig. 1d, lane 4-7). In particular, none of the alternate sDscam $\beta 1$ or $\beta 4$ isoforms formed aggregates. Moreover, the aggregates of cells expressing sDscam $\beta$ isoforms were smaller than those expressing sDscamas (Fig. 1d, Supplementary Fig. 1b). These observations suggest that the ability of each sDscam isoform to mediate homophilic aggregation differs in a cluster-specific manner.

Notably, cells expressing individual sDscam isoforms from the same cluster, which differed only in the $\mathrm{N}$-terminal variable region, exhibited markedly different cell aggregation activity. For example, sDscam $\beta 2 \mathrm{v} 6$ and $\beta 2 \mathrm{v} 8$ formed homophilic aggregates, but other members of the sDscam $\beta 2$ subfamily did not (Fig. 1d, lane 5). Similar results were obtained 
when assaying individually sDscam $\beta 3$ and $\beta 5$ isoforms (Fig. 1d, lane 6, 8). This discrepancy in the aggregation activity between isoforms from the same or different clusters was likely the consequence of differences in the expression, membrane localization, or intrinsic trans-binding affinities of individual sDscam isoforms ${ }^{23}$. Failure to form cell aggregates in mammalian Pcdh $\alpha$ isoforms is reportedly due to the lack of membrane localization ${ }^{23,35,36}$. However, this can be ruled out because immunostaining indicated that $\mathrm{sDscam} \beta 4 \mathrm{v} 3$ was present on the surface of Sf9 cells, as was sDscama14 (Supplementary Fig. 1c, panels i and iii). Therefore, we speculate that the different aggregation activities likely reflect, at least in part, differences in the intrinsic trans homophilic binding affinities of the individual isoforms.

\section{The first two N-terminal Ig domains are required for homophilic trans binding}

To explore how sDscam proteins mediated differential homophilic trans-binding, we firstly defined the minimum domain required for homophilic interactions. To this end, a series of $\mathrm{N}-$ terminal truncations of the extracellular domain of sDscama14 fused with mCherry were subjected to cell aggregation assay (Fig. 2a). We did not observe cell aggregation for each of these deletion constructs in which the first one to five domains were successively deleted from sDscama14 (Fig. 2a, panels ii-vi). Similarly, all constructs lacking the Ig1 domain did not show aggregation for sDscam $\beta 3 \mathrm{v} 3$ and $\beta 6 \mathrm{v} 1$ (Fig. 2a, panels viii-xii, Supplementary Fig. 2a, panels 2-6). Of all the truncated mutants in three independent assays, homophilic binding activity was dependent on the presence of the $\operatorname{Ig} 1$ domain (Fig. 2b). These results indicate that the first Nterminal domain $(\operatorname{Ig} 1)$ is required for sDscam-mediated homophilic trans binding.

We produced a series of truncations in which extracellular domains were successively 
deleted starting with the membrane-proximal FNIII3 domain (Fig. 2c). These truncations harboured one sDscam $\alpha$ and seven $\mathrm{sDscam} \beta$ isoforms, four of which were derived from two clusters, enabling comparison of various isoforms from the same cluster or from different clusters (Fig. 2c, Supplementary Fig. 2a). Representative examples of truncation are shown for sDscam $\alpha$, sDscam $\beta 1 v 2$, and $\beta 4 v 1$ in Fig. 2c. Five additional examples for sDscam $\beta 3 v 3, \beta 4 v 3$, $\beta 5 \mathrm{v} 10, \beta 6 \mathrm{v} 1$, and $\beta 6 \mathrm{v} 2$ are shown in Supplementary Fig. 2a. With the exception of the sDscam $\beta 1 \mathrm{v} 2$ truncated constructs, all of which did not induce cell aggregation as the complete ectodomains, at least one truncated construct containing the first two N-terminal Ig domains mediated cell aggregation for each sDscam tested (Fig. 2c, panels i-xviii, Supplementary Fig. 2a, panels 7-36), while all truncated constructs containing only the Ig1 domain did not (Fig. 2c, panels vi, xii and xviii, Supplementary Fig. 2a, panels 12, 18, 24, 30 and 36). These results indicate that the smallest protein that exhibited trans interaction contains two N-terminal Ig12 domains (Fig. 2c, d, Supplementary Fig. 2a), compatible with the fact that the variable Ig1 domain (possibly in combination with Ig2) acted as a trans-binding interface (see results below). Thus, the first two N-terminal Ig domains are essential for sDscam-mediated homophilic trans binding.

\section{Homophilic trans-binding is associated with the constant extracellular and}

\section{transmembrane domains of sDscam}

Based on data from eight independent assays with successive truncation from the membraneproximal extracellular domain (Fig. 2c, Supplementary Fig. 2a), we observed three different changes in aggregation. First, cell aggregation was gradually decreased as the number of domains deleted increased in most sDscam isoforms tested, such as $\mathrm{sDscam} \alpha 14, \operatorname{sDscam} \beta 5 \mathrm{v} 10$, 
$\beta 6 v 1$, and $\beta 6 v 2$ (Fig. 2c, panels i-vi, Supplementary Fig. 2a, panels 19-36). In these cases, constant domains might enhance sDscam-mediated homophilic trans binding. Second, deletion of two or three membrane-proximal FNIII domains in sDscam $\beta 4 \mathrm{v} 1$ and $\beta 4 \mathrm{v} 3$ rescued their cell aggregation activity, more efficiently in $\beta 4 \mathrm{v} 1 \Delta$ FNIII $1-3$ and $\beta 4 \mathrm{v} 3 \Delta$ FNIII1-3 (Fig. 2c, panels xiii-xviii, Supplementary Fig. 2a, panels 13-18). These observations suggest that the FNIII domains of sDscam $\beta 4$ inhibit homophilic trans binding. Third, we found that sDscam $\beta 1$ homophilic interactions could not be rescued by deletion of any domain (Fig. 2c, panels viixii). Therefore, the constant domains may be associated with homophilic binding in a clusterspecific manner.

Interestingly, the change in cell aggregation varies markedly upon domain truncation even between isoforms from the same cluster. For example, we observed cell aggregates in $\beta 6 \mathrm{v} 2 \Delta \mathrm{FNIII} 2-3, \beta 6 \mathrm{v} 2 \Delta \mathrm{FNIII} 1-3$, and $\beta 6 \mathrm{v} 2 \Delta \mathrm{Ig} 3-\mathrm{FNIII} 3$, but not in their $\beta 6 \mathrm{v} 1$ counterparts (Supplementary Fig. 2a, panels 27-29 and 33-35). Similarly, $\beta 4 v 3 \Delta$ Ig3-FNIII3, but not $\beta 4 \mathrm{v} 1 \Delta \operatorname{Ig} 3-\mathrm{FNIII} 3$, exhibited aggregation activity (Fig. 2c, panel xvii, Supplementary Fig. 2a, panel 17). This suggests that the constant domains influence homophilic trans binding by coupling with variable domains.

To further investigate how the constant extracellular domains contribute to homophilic trans binding, we performed experiments in which domains were shuffled between sDscama14, which binds, and sDscam $\beta 4 v 1$, which does not (Fig. 2e). Constructs in which the extracellular domain of sDscam $\alpha 14$ was replaced by the corresponding domain of sDscam $\beta 4 \mathrm{v} 1$, or vice versa, were produced and tested for cell aggregation activity (Fig. 2e, f). As a result, replacing the Ig2-FNIII3 or Ig3-FNIII3 constant domains of sDscam $\beta 4 \mathrm{v} 1$ with the corresponding region of 
sDscama14 caused homophilic binding, while chimeric constructs containing the FNIII1-3 or less-constant domains of sDscama14 did not show homophilic binding (Fig. 2e, f, panels i-vi). By contrast, replacing the FNIII1-3 or more-constant domains of sDscama14 by the corresponding region of $\mathrm{sDscam} \beta 4 \mathrm{v} 1$ failed to induce cell aggregation, while chimeric constructs containing the FNIII2-3 or FNIII3 domain of sDscam $\beta 4 \mathrm{v} 1$ resulted in cell aggregation (Fig. 2e, f, panels xi-Xvi). The fact that both truncated and shuffled constructs derived from sDscam $\beta 4 \mathrm{v} 1$ could mediate homophilic interactions indicates that there is no inherent physical barrier, such as binding incompatibility at the trans interface, preventing homophilic recognition of these proteins (Fig. 2c, panels xv and xvi, Fig. 2f, panel i and ii). Taken together, these data demonstrate that, in addition to matching between the N-terminal variable domains, the constant domains may affect homophilic trans binding.

To further determine which extracellular domain contributed to homophilic trans binding, we carried out chimeric mutation experiments in which a single domain was shuffled between sDscam $\alpha 14$ and sDscam $\beta 4 \mathrm{v} 1$. Replacement of the Ig3 or FNIII 1 domain of sDscam $\beta 4 \mathrm{v} 1$ by the corresponding region of sDscama14 caused detectable homophilic binding (Fig. 2f, panels viii and ix). Conversely, individual replacement of the Ig3-FNIII2 of sDscama14 by the corresponding domain of sDscam $\beta 4 \mathrm{v} 1$ decreased or even abolished homophilic binding activity (Fig. 2f, panels xvii-xix). Therefore, the Ig3 and FNIII1 domains play an important role in homophilic trans binding. Similar domain shuffling experiments were performed between sDscama14 and sDscamß1v1 (Supplementary Fig. 2b). However, individual or combined replacement of the constant extracellular domain of sDscam $\beta 1 \mathrm{v} 1$ by the corresponding region of sDscama14 did not cause detectable aggregation (Supplementary Fig. 2b, panels 3-7). Taken 
together, these results show that the constant extracellular domains contributed to regulating homophilic trans binding in a cluster-specific manner.

In addition, we performed domain-shuffling experiments between another sDscam pair: sDscama39 (very strong homophilic binding) and sDscam $\beta 3 \mathrm{v} 2 / \mathrm{v} 3$ (no or weak homophilic binding) (Fig. 2g, Supplementary Fig. 2c). These shuffling experiments not only identify the extracellular domain that contributes to the homophilic trans binding activity of a chimera but also indicate that the TM region is involved in homophilic binding (Fig. 2g, Supplementary Fig. 2c). Strikingly, replacement of the TM domain of sDscam $\alpha 39$ by that of sDscam $\beta 3$ significantly decreased homophilic binding activity (Fig. 2g, panels i-ii). Conversely, replacement of the transmembrane domain of sDscam $\beta 3 \mathrm{v} 2$ with the corresponding region of sDscam $\alpha 39$ caused detectable aggregation (Fig. $2 \mathrm{~g}$, panels iii-iv). Likewise, replacement of sDscam $\beta 3 \mathrm{v} 3$ with the transmembrane domain of sDscama39 significantly increased aggregation (Fig. 2g, panels vvi). However, replacement of sDscam $\beta 4 \mathrm{v} 1$ by the transmembrane domain of sDscam $\alpha 14$ did not affect its aggregation, and vice versa (Fig. 2e, f, panels vi versus $\mathrm{x}$, and $\mathrm{xvi}$ versus $\mathrm{xx}$ ). These results indicate that the transmembrane domain contributes to homophilic trans binding, at least in some sDscams.

Overall, the domain-swapping results, together with the domain-truncation experiments (Fig. 2a, c, Supplementary Fig. 2a), suggest that the extracellular and transmembrane domains might be involved in homophilic trans binding, with the relative contributions of each domain varying among the sDscam clusters. 


\section{sDscams exhibit highly isoform-specific binding}

To analyze the specificity of interaction between different sDscams as well as isoforms differing in the 5' variable Ig domains, we assessed cell aggregates formed by mixing two fluorescently labeled cell populations (Fig. 3a). Each sDscam was expressed with mCherry or enhanced green fluorescent protein (EGFP) fused to the C-terminus and assayed for binding specificity. We first investigated the specificity of the interaction between sDscam $\alpha$ isoforms, which differ in the Ig1 domain at the N-terminus. To determine the stringency of recognition specificity, we generated pairwise sequence identity heat maps of the variable Ig1 domains (Fig. 3b). Using these heat maps, we identified sDscam pairs with the highest pairwise sequence identity within their Ig1 domains. Fourteen of the closely related sDscams ( $>87 \%$ identity, Fig. 3b) were tested together with 21 more distantly related sDscams (Fig. 3c). In total, we tested 35 unique pairs of sDscams with sequence identity for non-self pairs ranging from $50-97 \%$ in the Ig1 domains. For most sDscam $\alpha$ pairs, only self-pairs on the matrix diagonals exhibited intermixing of red and green cells, while all non-self pairs formed separate, noninteracting homophilic cell aggregates (Fig. 3c-3e, Supplementary Fig. 3a). Identical results have been obtained for reciprocal binding pairs. These data indicate that the first variable Ig domain of sDscam $\alpha$ is sufficient to determine binding specificity.

However, heterophilic binding occurred between closely related variable domains. For example, sDscama20 and sDscama36 (96.8\% sequence identity in their Ig1 domains) showed remarkable heterophilic binding (Fig. 3f). A comparative analysis indicated that the thresholds for homophilic and heterophilic binding were $\sim 96 \%$ sequence identity in the Ig1 domains of sDscama pairs (Fig. 3g). We identified only one example of heterophilic binding among the 35 
Ig1 pairs. Thus, the vast majority of the sDscam $\alpha$ isoforms exhibited strict homophilic trans binding.

Next, we investigated the specificity of the interactions between sDscam $\beta$ isoforms, which differ in their Ig1-2 domains at the N-terminus. We generated pairwise sequence identity heat maps of the Ig1-2 domains and found that only one $\operatorname{sDscam} \beta$ pair shared more than $90 \%$ sequence identity between the Ig1 domains (Supplementary Fig. 3b). Because these isoforms did not support homophilic binding when individually assayed, we were unable to assess their binding specificity. In total, we tested 10 pairs of $\operatorname{sDscam} \beta / \beta$ and sDscam $\alpha / \beta$, respectively. All of the sDscam $\beta / \beta$ and $\alpha / \beta$ pairs tested bound strictly homophilically (Supplementary Fig. 3c, d). Taken together with the results of sDscama analysis (Fig. 3), these observations demonstrated a highly homophilic interaction between $\mathrm{sDscam} \alpha$ and $\mathrm{sDscam} \beta$ isoforms.

\section{Domain shuffling identifies variable Ig1 as key specificity-determining domains}

In contrast to $\mathrm{sDscam} \alpha \mathrm{s}, \mathrm{sDscam} \beta \mathrm{s}$ contain two variable Ig domains at the $\mathrm{N}$-terminus. To identify the variable domain responsible for the specificity of trans interactions between different $\mathrm{sDscam} \beta$ isoforms, we constructed a series of Ig-domain swapping chimeras between sDscam $\beta 5 \mathrm{v} 4$ and $\beta 5 \mathrm{v} 10$ isoforms within the same cluster (Fig. 4a). The two isoforms have $46.7 \%$ amino acid sequence identity within their variable Ig1 domains. As a result, a chimeric construct encoding sDscam $\beta 5 \mathrm{v} 4$ with its Ig1 domain replaced by that of sDscam $\beta 5 \mathrm{v} 10$ no longer interacted with its parent sDscam $\beta 5 \mathrm{v} 4$, but interacted with sDscam $\beta 5 \mathrm{v} 10$ (Fig. 4a, panels ii and iv, Supplementary Fig. 4b). By contrast, a chimeric construct encoding sDscam $\beta 5 \mathrm{v} 4$ with its Ig2 domain replaced by that of $\mathrm{sDscam} \beta 5 \mathrm{v} 10$ still interacted with its parent $\mathrm{sDscam} \beta 5 \mathrm{v} 4$, but 
not with sDscam $\beta 5 \mathrm{v} 10$ (Fig. 4a, panels i and iii). Identical results were obtained by Ig domain swapping between sDscam $\beta 5 \mathrm{v} 8$ and $\beta 5 \mathrm{v} 10$ (Fig. 4a, panels v-viii), and sDscam $\beta 5 \mathrm{v} 5$ and $\beta 5 \mathrm{v} 10$ (Supplementary Fig.4c). Therefore, the first Ig domain of sDscam $\beta$ is the primary determinant of trans interaction specificity.

Conversely, is a single common Ig1 domain sufficient to confer co-aggregation between sDscam $\beta$ isoforms? To determine this, we performed domain swapping between different sDscam $\beta$ subfamilies, which differ in both the variable and constant regions (Fig. 4b). A cell aggregation assay indicated that a chimeric construct encoding sDscam $\beta 6 \mathrm{v} 1$ with its Ig1 domain replaced by that of sDscam $\beta 5 \mathrm{v} 10$ interacted with $\mathrm{sDscam} \beta 5 \mathrm{v} 10$ (Fig. $4 \mathrm{~b}$, panel iv), and vice versa (Fig. 4b, panel viii). A similar result has been observed for domain swapping between sDscam $\alpha$ and sDscam $\beta$ subfamilies (Fig. 4c). In this case, cells expressing a chimeric construct encoding sDscam $\beta 3 \mathrm{v} 3$ with its Ig1 domain replaced by that of sDscam $\alpha 39$ co-aggregated with cells expressing sDscama39 (Fig. 4c, panel ii), and vice versa (Fig. 4c, panel viii). These domain swapping experiments between different sDscam subfamilies indicate that a single same Ig1 domain is sufficient for mediating trans binding specificity, at least for the sDscam isoform pairs tested. Conversely, these observations also showed that the constant region of sDscam might not be involved in defining its binding specificity. These data further support a key role for the Ig1 domain in determining the trans interaction specificity.

\section{sDscams interact in trans via antiparallel Ig1 self-binding}

To gain insight into how the variable Ig 1 domain mediates homophilic binding specificity, we carried out homology modeling studies to generate homodimeric complexes of sDscam $\alpha$ Ig1 
variants. Based on the crystal structure of variable $\operatorname{Ig} 7$ of fly Dscam1 ${ }^{37,38}$, we built an $\operatorname{Ig} 1$ homodimeric model of sDscam $\alpha 30$ with the SWISS-MODEL program and showed that sDscam $\alpha$ might adopt an antiparallel self-binding fashion of $\operatorname{Ig} 1 / \operatorname{Ig} 1$ (Fig. 5a) ${ }^{39,40}$. Furthermore, docking modeling of each sDscam $\alpha$ revealed that there was a complementary electrostatic potential surface pattern on the ABDE face: positive at one end and negative at the other (Fig. 5a). In this interface docking model, the positive residues might interact with neighboring negative residues to form a salt bridge in the ABDE face. For instance, residue 5 lysine $(\mathrm{K})$ (sDscama30 Ig1 numbering) in the A strand and residue 12 aspartic acid (D) in the AB loop region are in close structural proximity at the homophilic binding interface, and thus may form a salt bridge (Fig. 5a).

To confirm this, we performed single and double complementary mutations of these candidate residues and assessed their ability to mediate cell aggregation. As a result, a single $\mathrm{K}$ mutation of residue 5 in the $\mathrm{A}$ strand or a $\mathrm{D}$ mutation of residue 12 in the $\mathrm{AB}$ loop region weakened cell aggregation (Fig. 5b, panel ii and iii). These results indicate that residue K5 in the A strand and residue D12 in the AB loop region are necessary for efficient self-recognition of sDscama30. Interestingly, double K/D mutants (K5D; D12K), which are thought to reform a salt bridge at the interface, partially restored cell aggregation (Fig. 5b, panel iv), validating an antiparallel self-binding mode of $\operatorname{Ig} 1 / \operatorname{Ig} 1$. Therefore, the $\operatorname{Ig} 1$ domains of sDscam $\alpha$ adopt an antiparallel self-binding conformation.

\section{Identification of sDscam Ig1 specificity-determining residues}

We next identified the Ig1 specificity-determining residues by structural modeling and 
mutagenesis studies. Candidate specificity-determining residues were predicted using the closely related Ig1 of sDscam (Supplementary Fig. 5a). Because the homophilic binding specificity of fly Dscam1 Ig7, a Chelicerata sDscam Ig1 homologue, is largely determined by electrostatic and symmetry axis residues 37,38 , different residues between each pair were screened based on their electrostatic and shape complementarity. A remarkable example is the closely related $\alpha 20 / \alpha 36 / \alpha 30$ pairs, in which sDscama36 exhibited robust heterophilic binding to sDscama20 but no heterophilic binding to sDscama30 (Fig. 3f). Four amino acids were different between the Ig1 domains of $\alpha 30$ and $\alpha 36$; however, only residue 22 of the B sheet, which resided at the symmetry axis, was predicted to influence shape complementarity (Fig. $5 \mathrm{c}$, panel i). Therefore, we speculate that this residue is a candidate specificity determinant.

To confirm this, we swapped them between isoforms and examined the binding specificities of the swapped isoforms and their parents (Fig. 5c, panel ii). Swapping residue 22(T/I), but not the other three residues $(1 \mathrm{~S} / \mathrm{P}, 15 \mathrm{~S} / \mathrm{N}$, and $21 \mathrm{~V} / \mathrm{I})$, switched the trans binding specificity between sDscama30 and sDscama36 (Fig. 5c, panel ii and iii). Cells expressing a swapped sDscama30 isoform in which residue 22T was replaced with 22I from sDscama36 and sDscama20 intermixed with cells expressing sDscama36 and sDscam $\alpha 20$ (Fig. 5c, panel iii). By contrast, these cells segregated from those expressing the parent isoform from which the swapped residue derived, and vice versa. Thus, a single residue is sufficient to determine binding specificity in some sDscam $\alpha$ pairs.

However, swapping residue $22(\mathrm{I} / \mathrm{T})$ of $\operatorname{Ig} 1$ between sDscam $\alpha 11$ and $\alpha 15$, or between sDscam $\alpha 13$ and $\alpha 15$, did not swap binding specificity but instead produced novel homophilic binding specificity (Supplementary Fig.5c). These observations suggest that additional residues 
at the $\operatorname{Ig} 1 / \operatorname{Ig} 1$ interface also contribute to binding specificity. By swapping interface residues that differed between other pairs of Ig1 variants (sDscama11 and $\alpha 13 ; \alpha 23$ and $\alpha 27$ ), the symmetry axis residue $52(\mathrm{M} / \mathrm{V} ; \mathrm{L} / \mathrm{V})$ of the $\mathrm{D}$ sheet was shown to alter, but not swap, the binding specificity (Supplementary Fig.5e). In the latter case ( $\alpha 27 / \alpha 23)$, separate green and red homophilic aggregates are formed, but they now adhere to one another (Supplementary Fig.5e, panel 18 and 20). By contrast, swapping residue 26 (N/I) between sDscama11 and $\alpha 13$, which was neither electrostatic nor located at the symmetry axis, did not alter the binding specificity (Supplementary Fig.5d). Collectively, these data indicate that residues 22 of the B sheet and 52 of the D sheet act as specificity determinants, at least for some pairs of sDscam isoforms.

Another representative example of specificity swapping is shown for the $\alpha 21 / \alpha 37$ pair (Fig. 5d). In the $\alpha 21 / \alpha 37$ pair, three different residues $(5,10 \& 56)$ were filtered as candidate specificity determinants, one of which interacted at the antiparallel self-binding interface by double complementary mutations (Fig. 5b). We found that swapping one of the three different residues $(5,10 \& 56)$ between sDscam $\alpha 21$ and $\alpha 37$ did not swap binding specificity (Fig. $5 \mathrm{~d}$, panel iii, Supplementary Fig. 5g). By contrast, swapping of all three residues of sDscama21 to those of sDscama37 swapped the binding specificity (Fig. 5d, panel iii). Conversely, swapping two of the three residues partially or completely altered the binding specificity between sDscama21 and $\alpha 37$ (Fig. 5d, Supplementary Fig. 5g). Similarly, swapping of all three residues 6, 19 and 52 between sDscama23 and $\alpha 27$ fully switched the binding specificity (Supplementary Fig. 5f). Therefore, these residues are involved in determining binding specificity. This may also, at least in part, mirror Ig1 diversification during sDscam evolution, where initial mutations after exon duplication could lead to promiscuous binding and additional 
combinatorial mutations could eventually produce a highly specific homophilic interaction distinct from their parents.

In summary, by structural modeling and mutagenesis studies, we identified specificitydetermining residues for sDscam isoforms (Supplementary Fig. 5b). These residues frequently resided at the center or side-chain of symmetric homo-dimerized interfaces, which exhibit electrostatic and shape complementarity between the ABED interface strands. As the six pairs of variable Ig domains used in these residue swapping experiments are not closely related (Supplementary Fig.5a), these interface residues likely contribute to determining homophilic specificity for other sDscam isoforms as well.

\section{sDscams form high order cis-multimers independent of their trans interactions}

Having established that sDscam isoforms are mediated by an $\operatorname{Ig} 1 / \operatorname{Ig} 1$ antiparallel self-binding between apposed cell surfaces, we attempted to explain how sDscam isoform diversity mediates self-recognition in Chelicerata. As estimated by the number of Ig1 or its orthologues, the number of Dscam isoforms is in the range of $\sim 100$ across the Chelicerata species ${ }^{32}$, at least two orders of magnitude lower than that in flies. Moreover, as shown above (Fig. 1), almost half of sDscam isoforms, when expressed individually, failed to engage in homophilic interactions in the cell aggregation assay (Fig. 1d). Therefore, the small number of sDscam isoforms in Chelicerata alone cannot account for its non-self-discrimination as for the homophilic interaction model in Drosophila Dscams. Thus, how do these nonclassical sDscam isoforms mediate homophilic interactions? Given the striking organizational resemblance 
between the scorpion $s D$ scams and mammalian $P c d h$, we speculate that via $c i s$-multimers scorpion sDscams function as vertebrate-clustered Pcdh isoforms ${ }^{22,23,29}$.

To confirm this hypothesis, we first performed coimmunoprecipitation (co-IP) analyses to investigate the interactions between sDscam isoforms in vitro (Fig. 6a). When HAsDscam $\beta 6 \mathrm{v} 2$ and Myc-sDscam $\beta 6 \mathrm{v} 2$ are coexpressed by coinfecting with individual recombinant viruses, Myc-tagged proteins could strongly coimmunoprecipitate with HA- $\beta 6 \mathrm{v} 2$ (Fig. 6b). Further co-IP experiments indicate that sDscam proteins from different subfamilies tested interacted strongly with each other (Fig. 6b, Supplementary Fig. 6a), exhibiting no specificity between different isoforms. Because sDscam $\alpha \Delta \operatorname{Ig} 1 \Delta \mathrm{TM}$ could interact with other sDscam $\Delta \operatorname{Ig} 1$ or sDscam $\Delta \operatorname{Ig} 1-2$ mutants (Supplementary Fig. 6b), in which Ig1-domain deletion has ablated homophilic trans interactions, robust co-IP between HA-sDscam and Myc-sDscam should not result from trans interactions, but cis interactions. These results demonstrate that sDscams interacted with each other in cis with almost no specificity between different isoforms.

To further characterize the cis interaction between sDscam isoforms, we performed multimer analysis by western blotting in the absence or presence of reducing agents. In reducing SDS/PAGE gels, sDscam $\beta 6 \mathrm{v} 2$ migrated with a single molecular weight of $\sim 80 \mathrm{kDa}$, which corresponded to the size of the monomer (Fig. 6c). Under non-reducing conditions, however, several large bands migrated behind the monomer, which corresponded to the size of sDscam assembly of putative dimer, tetramer, and larger multimers (Fig. 6c). Notably, all sDscam assembly sizes were a multiple of the dimer, suggesting that the dimer acts as a basic recognition unit for sDscam, and clusters into tetramer and higher order oligomeric complexes. 
Similarly, we performed multimerization analysis by expressing proteins of all other sDscam $\alpha$ and sDscam $\beta 1-\beta 5$ subfamilies. We observed multimerization to different extents in all sDscam proteins investigated, suggesting that sDscam proteins from different subfamilies are able to homo-multimerize (Fig. 6d, panel i). In these cases, we found that individual sDscam isoforms from the same cluster (i.e., sDscama1, $\alpha 14, \alpha 25)$ exhibited largely similar multimerization behavior, which varied markedly among the sDscam proteins from different subfamilies. Furthermore, we have observed abundent endogenous sDscam $\beta 6 v 2$ multimers in the cephalothorax of scorpion (Fig. 6d, panel ii). Taken together, our results demonstrate that sDscams can form strong multimers in vivo.

It is possible that sDscam multimers result from trans interactions in an antiparallel orientation (Fig. 6a). However, sDscam $\beta 1 \mathrm{v} 2$ and $\beta 4 \mathrm{v} 1$, which did not show homophilic trans interactions in cell aggregation assays, still exhibited robust multimers (Fig. 6d, panel i). Moreover, Ig1-2 domain deletions, which ablated homophilic trans interactions, still exhibited strong multimerization (Fig. 6e, panel i). Therefore, sDscam cis interactions occurred even in the absence of trans interactions. To preclude the effect of sDscam size on multimerization, we performed single amino acid mutations in the Ig1 domain of sDscams and examined the effect on multimerization (Fig. 6e, panel ii). Single mutations, which disrupted homophilic trans interactions in a cell aggregation assay, heightened multimerization (Fig. 6e, panel ii). These results support the notion that sDscams multimerized in cis independently of their trans interactions and indicate that robust sDscam multimers result from cis interactions. 


\section{sDscam cis multimerization is mediated by the extracellular FNIII1-3 domains}

To elucidate how sDscams form high order cis-multimers, we constructed a series of truncations of the extracellular domain of sDscam $\beta 6 \mathrm{v} 2$ fused with a Myc-tag for cis multimerization assay by sequentially deleting the domain from the N-terminus (Fig. 6f). We found that the truncated proteins lacking Ig1 ( $\beta 6 \mathrm{v} 2 \Delta \operatorname{Ig} 1)$, Ig1-2 ( $\beta 6 \mathrm{v} 2 \Delta \operatorname{Ig} 1-2)$, and Ig1-3 ( $\beta 6 \mathrm{v} 2 \Delta \operatorname{Ig} 1-3)$ exhibited robust multimerization (Fig. 6f, panel i, lane 2-4). Notably, the truncated proteins lacking Ig1, Ig1-2, and Ig1-3 exhibited multiple bands, which corresponded to the size of sDscam assembly putative dimers, tetramer, hexamer, octamer, and larger multimers as the entire extracellular domain, respectively (Fig. 6f, panel i). Therefore, the absence of Ig1, Ig1-2, and Ig1-3 did not influence the assembly pattern of multimerization. Importantly, based on the unaltered assembly pattern of multimers in a series of successively truncated proteins, we reason that sDscams are assembled into cis-dimer and higher-order multimeric complexes in a parallel orientation (Fig. 6f, panel ii). Furthermore, sDscam mutants lacking Ig1-2 or Ig1-3 exhibited the most heightened cis multimerization (Fig. 6f, arrows in panel i). Together, these data strongly suggest that the Ig1-3 domains of sDscam are dispensable for $c$ is interactions, and that membrane-proximal FNIII1-3 domains are sufficient for efficient cis multimerization.

However, when reduced to one or two membrane-proximal FNIII domains, the resulting constructs (i.e, $\beta 6 \mathrm{v} 2 \Delta \mathrm{Ig} 1-\mathrm{FNIII} 1$ and $\beta 6 \mathrm{v} 2 \Delta \mathrm{Ig} 1-\mathrm{FNIII} 2)$ exhibited markedly reduced multimerization (Fig. 6f, lane 5, 6). Because these constructs contained the same transmembrane domain, the discrepancy between them is likely due to the presence of different FNIII domains. To further identify the extracellular domains of sDscam that contribute to cis multimerization, we constructed mutants encoding a single or two continuous extracellular 
domains with an HA tag and co-transfected them with sDscam $\beta 6 v 2$ (Fig. 6g). Co-IP experiments revealed that the deletion constructs containing individual or multiple continuous FNIII domains were capable of binding to sDscamß6v2 (Fig. 6g, panel i, Supplementary Fig. 6c), indicating that sDscam $\beta 6 \mathrm{v} 2$ can interact with each FNIII domain. This observation was supported by cis multimerization assays, which showed that each of the truncated $\beta 6 \mathrm{v} 2$ proteins could form strong cis-multimers (Fig. 6g, panel ii). These data indicate that cis multimerization is jointly mediated by all three membrane-proximal FNIII1-3 domains. This result is also consistent with computational modeling using the ZDOCK server ${ }^{41}$, by which sDscam $\beta 6 \mathrm{v} 2$ could form a homodimer via multiple parallel interfacial regions involving the FNIII1-3 domains (Supplementary Fig. 6d). Because the sDscam $\beta 6$ v2 FNIII1-3 domains lack cysteine residues, they likely mediate cis multimerization by noncovalent mechanisms.

We subsequently carried out multimerization assays of all other sDscam $\alpha$ and sDscam $\beta 1-$ $\beta 5$ proteins by truncating the extracellular domain as for sDscam $\beta 6 \mathrm{v} 2$. Representative examples of multimerization truncation are shown for sDscam $\alpha 14$, sDscam $\beta 1 \mathrm{v} 2$, sDscam $\beta 2 \mathrm{v} 6$, and sDscam $\beta 5 v 2$ in Supplementary Fig. 6e. Consistent with sDscam $\beta 6 v 2$, the absence of the Nterminal Ig1-3 domains increased the extent of cis multimerization in almost each sDscam (arrows in graph of Supplementary Fig. 6e). These observations suggest that the membraneproximal FNIII1-3 domains of sDscams are sufficient for efficient cis multimerization. However, when deleting the FNIII domains, individual sDscams from different subfamilies exhibited different patterns of alteration of multimerization (Supplementary Fig. 6e). For instance, the FNIII2-3 peptide (i.e, $\beta 5 \mathrm{v} 2 \Delta \mathrm{Ig} 1-\mathrm{FNIII} 1)$ exhibited similar multimerization to the FNIII1-3 peptide ( $\beta 5 \mathrm{v} 2 \Delta \operatorname{Ig} 1-3)$ in sDscam $\beta 5 \mathrm{v} 2$ (Supplementary Fig. 6e, panel iv, lane 4,5$)$, 
while the FNIII2-3 peptide (i.e, $\beta 6 \mathrm{v} 2 \Delta \operatorname{Ig} 1-\mathrm{FNIII} 1)$ exhibited much less multimerization than the FNIII1-3 peptide ( $\beta 6 \mathrm{v} 2 \Delta \operatorname{Ig} 1-3)$ in sDscam $\beta 6 \mathrm{v} 2$ (Fig. 6f, panel i, lane 4,5$)$. This suggests that the contribution of individual FNIII domains to cis interactions differs markedly among sDscams. Taken together, our results indicate that all three membrane-proximal FNIII1-3 domains engage in cis multimerization of sDscams.

In summary, our data reveal that all three membrane-proximal FNIII1-3 domains engaged in high order cis multimerization, while the first N-terminal Ig1-3 domains were dispensable for cis interactions in all sDscams investigated. Notably, despite their general promiscuity, all FNIII1-3 domains of sDscam may contribute to $c$ is interactions, with the relative contributions of each domain to cis multimerization varying from one sDscam cluster to another.

\section{Transmembrane domain promotes the formation of sDscam cis-multimers}

We subsequently investigated whether and how the transmembrane (TM) domain of sDscam contributes to high-ordered cis multimerization. To this end, we first examined how TM deletion affected self-multimerization of sDscam. Each of the truncated proteins lacking the TM domain was capable of multimerizing, indicating that the extracellular domain is sufficient to confer efficient cis multimerization (Fig. 6h, Supplementary Fig. 6f). However, the multimerization efficiency was markedly reduced in most sDscam $\Delta \mathrm{TM}$ mutants (arrows in Fig. 6h, Supplementary Fig. 6f). For example, dimer and tetramer were reduced in sDscam $\alpha \Delta T M$, while tetramer was almost undetectable in $\beta 6 \mathrm{v} 2 \Delta \mathrm{TM}$ (Fig. 6h, panel i). Overall, these data demonstrate that although the extracellular region alone might engage in the formation of multimers in cis, the presence of the transmembrane domain greatly enhances the accuracy and 
efficiency of sDscam cis multimerization.

To further determine the role of the TM domain in cis multimerization, we examined whether sDscam transmembrane peptides, in the absence of regulation by soluble domains, have the propensity for self-multimerization. When the expressed transmembrane peptides were treated in the absence or presence of reducing agents, we observed dimerization of the transmembrane peptides expressed from all six sDscams investigated (Fig. 6h, panel ii), while the addition of reducing agents precluded dimer formation. These observations indicate that the presence of the transmembrane domain alone is sufficient to confer efficient cis dimerization in each sDscam. However, we have not observed higher order multimers (i.e., tetramer, hexamer) for sDscam transmembrane peptides as extracellular proteins. Notably, although individual transmembrane peptides from all sDscams engaged in dimerization, the efficiency of dimerization varied remarkably among them, with the greatest efficiency for sDscam $\alpha$ and sDscam $\beta 6$ and the least for sDscam $\beta 4$ and sDscam $\beta 5$ (Fig. 6h, panel ii). By contrast, TM peptides from sDscam $\beta 4$ and sDscam $\beta 5$ accounted for less than $10 \%$ of dimers (Fig. 6h, panel ii). These results further implicate the transmembrane domain in sDscam cis multimerization.

To further explore how the transmembrane domain promotes sDscam cis multimerization, we analyzed the roles of transmembrane residues using SWISS-MODEL ${ }^{39,}{ }^{40}$. As expected from sequence-based $\alpha$-helical transmembrane prediction tools, several of the residues in the membrane form a contiguous helix in sDscam $\beta 6 \mathrm{v} 2$, which have a propensity to dimerize via covalent or noncovalent interactions (Supplementary Fig. 6g, panel i). Notably, the transmembrane of some sDscams (i.e, $\mathrm{sDscam} \beta 2, \beta 3$ ), which did not contain cysteine residues, exhibited considerable dimerization (Fig. 6h, panel ii). This observation suggests that disulfide 
bonds within the transmembrane domain are not essential for cis dimerization at least in some sDscams. A further mutation experiment showed that cysteine residue mutation in the transmembrane domain of sDscam $\beta 6 \mathrm{v} 2$ did not markedly affect the formation of cis-multimers in both the $\beta 6 \mathrm{v} 2$ and $\beta 6 \mathrm{v} 2 \Delta \operatorname{Ig} 1-3$ constructs (Supplementary Fig. $6 \mathrm{~g}$, panel ii). Collectively, these results demonstrate that the transmembrane domain of each sDscam mediated cis multimerization, likely via a noncovalent mechanism.

\section{Coexpression of multiple sDscam isoforms diversify homophilic specificities}

Finally, because all sDscam $\alpha$ isoforms and some of $\operatorname{sDscam} \beta s$ mediated homophilic trans bindings (Fig. 1d) and interacted with each other in cis without specificity (Fig. 6), we tested how combinatorial expression of multiple sDscam isoforms diversified binding specificities. In all cases, cells coexpressing two sDscam $\alpha$ combinations failed to coaggregate with cells expressing two different sDscam $\alpha$ combinations. However, intermixed cell aggregates of cells that coexpressed identical sDscam $\alpha$ combinations were observed (Fig. 7a). Consistent with these observations, co-IP experiments showed different sDscamas interacted strongly with each other when coexpressed (Supplementary Fig. 7b, lane1-2). Similar data were obtained for each of the sDscam $\alpha / \beta$ pairs (Fig. 7b, Supplementary Fig. 7b, lane 3-4). These results suggest that homophilic specificity by coexpressing two distinct isoforms depends on the identity of the isoform pair but not a single isoform. These results strongly suggest that sDscams interact in cis so as to create new homophilic specificities that differ from the specificities of the individual sDscam isoforms. 
To further examine combinatorial homophilic specificities, we coexpressed distinct sets of three sDscam isoforms, and then evaluated their ability to coaggregate with cells containing various numbers of mismatches (Fig. 7c). In all cases, only cells expressing identical isoform combinations formed intermixed coaggregates, while cells expressing mismatched isoforms displayed separate red and green aggregates (Fig. 7c). Remarkably, even a single isoform common between cells coexpressing three isoforms and expressing one isoform caused separate red and green aggregates (Fig. 7c, panels 8-10). Taken together, these results strongly suggest that sDscam isoforms evolved a unique combinatorial multimerization to diversify homophilic specificities.

\section{Discussion}

Here, we provide compelling evidence that different combinations of sDscam isoforms interact in cis to significantly expand homophilic trans recognition specificities in Chelicerata. Specifically, we showed that sDscam isoforms form promiscuous cis-multimers involving the membrane-proximal FNIII1-3 and transmembrane domains at the cell surface that associate specifically in trans via $\operatorname{Ig} 1 / \operatorname{Ig} 1$ self-binding. Thus, our data revealed that Chelicerata sDscams mediated highly isoform-specific homophilic interactions via a "hybrid" mechanism between fly Dscam1 and vertebrate Pcdhs. Below, we discuss the molecular basis of the homophilic interactions of sDscam isoforms, and the implications of sDscam architecture in neuronal selfrecognition and non-self-discrimination, with particular emphasis on comparison to fly Dscam1 and vertebrate Pcdhs.

\section{Chelicerata sDscams form high order cis-multimers}


Our data clearly indicate that sDscam isoforms could form robust cis-multimers even in the absence of trans interactions (Fig. 6). This is supported by several lines of independent evidence that distinct sDscam isoforms from different clusters can be coimmunoprecipitated (Fig. 6b, Supplementary Fig. 6a), and that sDscams are present in high-molecular-weight, detergentsolubilized assembly complexes from scorpion cephalothorax (Fig. 6d, panel ii). In addition, we observed altered recognition specificity when multiple sDscam isoforms were coexpressed (Fig. 7a-c), further supporting their cis multimerization. Such cis multimerization of Chelicerata sDscams is in sharp contrast to that of fly Dscam1, which appear interacts as cis monomers. Although the individual orthologue of FNIII1-3 domains in Chelicerata sDscams is present at the membrane-proximal region of fly Dscam1, the overall structure of the membrane-proximal region might be reconstituted in evolutionary transitions from classical Dscam to shortened sDscams. Because sDscam originated from classical Dscam after Chelicerata speciation ${ }^{14,33}$, sDscam cis multimerization might be in large part the result of evolutionary co-adaptation of these membrane-proximal domains as a cis interaction interface for combinatorial recognition specificity.

Although Chelicerata sDscams form cis-multimers like Pcdh isoforms, they differ in at least two major aspects. Firstly, Chelicerata sDscams form $c i s$-multimers via a $c i s$ interaction interface distinct from Pcdhs. The latter form cis dimers mediated by membrane-proximal EC6 or both EC5 and EC6 ${ }^{23,24,25,29}$. By contrast, the formation of sDscam cis-multimers might be mediated by combining membrane-proximal FNIII1-3 and transmembrane domains (Fig. 6fh). Although individual recognition sites located in the extracellular and transmembrane domains alone might engage in the formation of cis- multimers, a long-range cis interface 
encompassing the FNIII1 to transmembrane domains could assure the accuracy and efficiency of cis multimerization. Thus, the sDscam cis interface involves a much larger proportion of the extracellular region than vertebrate Pcdhs. This discrepancy in the cis interface may at least in part account for the markedly different cis multimerization ability of Pcdhs and sDscams, whereas a $\gamma$-Pcdh lacking EC1-3 domains interacted weakly ${ }^{22}$ while sDscams lacking Ig1-3 domains exhibited robust cis multimerization for the one sDscam $\alpha$ and six sDscam $\beta$ subfamilies tested (Fig. 6, Supplementary Fig. 6).

Furthermore, sDscams formed more complex cis-multimers than Pcdhs. Although $\gamma$ Pcdhs reportedly form cis tetramers ${ }^{22}$, it is generally recognized that Pcdhs form cis-dimers, as evidenced by dimerization of truncated Pcdhs lacking the EC1 or EC1-2 domain in solution ${ }^{25,}$ ${ }^{29}$. By contrast, our evidence showed that sDscams assembled into cis-dimer, tetramer, and even higher-order oligomeric complexes (Fig. 6, Supplementary Fig. 6). Therefore, sDscams seem to exhibit more efficient and complex cis multimerization than Pcdhs, presumably due to their larger interaction interface.

\section{sDscams mediate highly specific homophilic recognition via self-binding variable Ig1}

We showed that all sDscam $\alpha$ isoforms can mediate homophilic interactions (Fig. 1d, 3b-f, Supplementary Fig. 3a), while a majority of sDscam $\beta 1-\beta 6$ isoforms does not interact homophilically (Fig. 1d). However, sDscam $\beta$ chimeric constructs (i.e, $\operatorname{sDscam} \beta 4 \mathrm{v} 1$ ) produced by deleting or replacing their partial constant region mediated homophilic interactions (Fig. 2c, panels xv and xvi; Supplementary Fig. 2a, panel 15-17; Fig. 2f, panel i and ii), indicating that the failure in homophilic recognition is not due to incompatibility of the trans self-binding 
interface. Thus, it seems likely that at least some of these sDscam $\beta$ s mediate self-recognition, although the mechanism is not yet understood. Alternatively, because sDscam cis-multimer assembly resembles the structures of antibody and T-cell receptor (TCR) in vertebrate, both comprising $\mathrm{N}$-terminal variable Ig domains and a C-terminal constant domain, it is likely that scorpion sDscam isoforms participate in heterophilic binding with other proteins or pathogens as vertebrate antibody and TCR. Moreover, $\sim 100$ sDscam isoforms in scorpion could potentially form a repertoire of $10^{8-12}$ structurally variable assemblies, which is compatible with the order of magnitude of the diversity of antibodies in vertebrates. Notably, pancrustacean Dscam1 isoforms play an immune-protection role in bacterial challenge $42,43,44$. Given the extraordinary diversity and high structural similarity between Chelicerata sDscam and vertebrate antibody and TCR, it is attractive to speculate that sDscam diversity plays a role in invertebrate immunity.

Based on structural modeling and mutagenesis experiments, we demonstrate that sDscam homophilic specificity is determined by an antiparallel $\operatorname{Ig} 1 / \operatorname{Ig} 1$ self-binding (Fig. 5a, b). Because the Ig1 domain of Chelicerata sDscams is orthologous to the $\operatorname{Ig} 7$ of fly Dscam1, it is reasonable that they have an identical antiparallel self-binding architecture. Indeed, sitedirected swapping mutagenesis revealed that Ig1 of Chelicerata sDscams shared several key specificity-determining residues with $\operatorname{Ig} 7$ of fly Dscam1 isoforms ${ }^{37,} 38$ (Fig. 5c, d, Supplementary Fig. 5). However, the homophilic specificity of fly Dscam1 is determined via three independent antiparallel self-binding modules, $\operatorname{Ig} 2 / \operatorname{Ig} 2$, $\operatorname{Ig} 3 / \operatorname{Ig} 3$, and $\operatorname{Ig} 7 / \operatorname{Ig} 7$ (Fig. 8) ${ }^{37,38}$, which can assemble in different combinations to generate a repertoire of tens of thousands of self-binding interfaces. By contrast, $\operatorname{Ig} 1 / \operatorname{Ig} 1$ self-binding of Chelicerata sDscams only 
generated $\sim 100$ distinct trans interfaces. If Chelicerata sDscams use the same neuronal selfrecognition mechanism as fly Dscam1, this number is not sufficient to discriminate self from non-self ${ }^{11}$. Therefore, trans homophilic interactions in Chelicerata sDscams likely proceed via a mechanism distinct from that of fly Dscam1.

By contrast, sDscam-mediated self-recognition is analogous to that of Pcdhs, which is mediated by a mechanism coupling cis and trans interactions ${ }^{22,23,24,25,29}$. In vertebrate Pcdhs, ectodomains recognize each other via variable EC1/EC4 and EC2/EC3 trans homophilic interactions $23,24,25,26,27,28,29$. Compared with the four EC domain-mediated trans interfaces in Pcdhs, the Chelicerata sDscam recognition interface contains only one Ig1 domain (or possibly with Ig2). Considering the fact that sDscams have a smaller proportion of trans interfaces, albeit with a larger cis interface, than Pcdhs, we speculate that stronger cis multimerization of sDscams compensates for their smaller trans adhesive interface to facilitate formation of stable cis/trans assembly complexes.

\section{Proposed model of sDscam interactions in self-non-self discrimination}

Based on a large body of experimental evidence and structural modeling, we propose that sDscams form promiscuous cis-multimers at the cell surface that associate specifically in trans via an independent Ig1 self-binding interface. Similar to mouse Pcdhs $24,25,27,28,29$, it is likely that full-length sDscam ectodomains in solution form a discrete array of multimers through specific trans dimerization of cis multimeric recognition units. However, as in Pcdhs ${ }^{23,29}$, such a structure cannot explain neuronal self-non-self discrimination because neurons with many neurites encounter insufficient diversity for self-recognition. Obviously, this self-recognition 
problem would be more severe for Chelicerata sDscams, which formed higher-order multimers than Pcdhs. Therefore, we speculate that Chelicerata sDscams could not adopt a discrete trans dimer of a cis multimeric assembly, but a zipper-like structure coupling cis and trans interactions like mouse Pcdhs 24, 25, 26, 27, 28, 29.

However, in contrast to Pcdh cis-dimeric recognition units $24,25,26,27,28,29$, sDscams can form cis tetramers and higher order multimers (Fig. 7d). Therefore, using sDscam cis tetramers as an example, we propose a two-dimensional latticed assembly structure model to account for sDscam-mediated cell-cell recognition (Fig. 7e). In this model, each sDscam cis tetramer could interact with multiple $c$ is tetramers on apposed cell surfaces via independent trans Ig1/Ig1 selfbinding, thereby forming a connected latticed assembly of proteins between cells. This model seems to be a "hybrid" structural framework between fly Dscams and vertebrate Pcdhs for Chelicerata sDscams, in which the $\operatorname{Ig} 1 / \operatorname{Ig} 1$ self-binding trans interface is analogous to that of Ig7 of fly Dscams, while sDscam membrane-proximal cis-multimeric interfaces tended to largely resemble to that of vertebrate Pcdhs.

This model provides a reasonable explanation for how neurites discriminate self from non-self in Chelicerata, although detailed structures are not available. The presence of identical sDscam isoforms in two neurites of the same neuron would enable individual Ig1s of cismultimers on apposed cell surfaces to self-bind each other independently of the composition of cis-multimers. Thus, the trans dimerization of cis-multimers could lead to a dense and connected lattice assembly between two apposing cell-surfaces, triggering strong homophilic interactions and inducing neurite repulsion (Fig. 7f). By contrast, because two neighboring neurons are incapable of expressing the same set of sDscam isoforms, mismatched Ig1s would 
lead to a scattered or sparse connected lattice assembly between apposing cell surfaces, triggering weak homophilic interactions. Thus, the resulting downstream signaling is below the threshold level and fails to initiate neurite repulsion (Fig. 7f). This model provides an evolutionary rationale for the smaller isoform number in Chelicerata by at least two orders of magnitude than that in flies. Although there are only $\sim 100$ distinct sDscam isoforms in Chelicerata ${ }^{32}$, this vast repertoire of combinatorial recognition specificities is sufficient to provide each neuron with a unique identity to discriminate between self and non-self. The major challenge for future studies will be to develop genetic techniques for Chelicerata species through which the sDscam diversity in the nervous system can be artificially manipulated.

\section{Chelicerata sDscams show more parallels with vertebrate Pcdhs than Drosophila Dscam1}

Our findings indicate that Chelicerata sDscams have striking parallels with Drosophila Dscam1 and vertebrate $P c d h$ s, suggesting analogous roles (Fig. 8). Three encode large numbers of neuronal transmembrane protein isoforms; the individual isoforms are expressed stochastically and combinatorially, and the encoded proteins interact homophilically (Fig. 8) $2,3,14$. In addition, such striking isoform diversity appears to underlie neuronal self-non-self discrimination, at least for the well-characterized Drosophila Dscam1 and vertebrate Pcdhs 11, 12, 30, 31, 45. Our results support and extend the notion that different phyla used different molecules or mechanisms to underlie analogous principle for mediating self-recognition and self-avoidance during neuronal arborization (Fig. 8) ${ }^{1,2}$. It would be interesting to determine which molecules mediate self-avoidance in other invertebrate phyla in the evolutionary gap between arthropods 
and vertebrates, particularly those lacking extensive Pcdh or Dscam diversity (e.g., the lancelet Branchiostoma floridae).

From an evolutionary viewpoint, Chelicerata sDscams are closely related with Drosophila Dscam1, but are not related with vertebrate Pcdhs ${ }^{3,33}$. However, in many respects, Chelicerata sDscams have more parallels with vertebrate Pcdhs (Fig. 8). Both are organized in a tandem array in the 5' variable region, encoding the same order of magnitude of isoforms $(50 \sim 100)$ via alternative promoters. Both have a similar structural composition comprising six extracellular domains, a single transmembrane domain, and a cytoplasmic region. We have now shown that scorpion sDscam, like mouse Pcdhs, exhibited combinatorial recognition specificities based on the assembly of cis-multimeric recognition units, thereby sharing similar neuronal self-recognition logic with vertebrate Pcdhs. Thus, our findings further blur the distinction between the self-avoidance of invertebrates and vertebrates. It will be interesting to learn if convergent examples for self-avoidance in other animals are available. Finally, based on the remarkable parallels between Chelicerata sDscams and vertebrate Pcdhs, we wonder whether cadherins, in the animal kingdom, generate extraordinary isoform diversity via alternative splicing like their fly Dscam1 counterparts. One thing is certain-insight from extraordinary isoform diversity continues to deepen our understanding of basic biological principles. 


\section{Methods}

\section{Cell lines}

Sf9 cells (a gift from Jian Chen, Zhejiang Sci-Tech University) were cultured in Sf-900 ${ }^{\mathrm{TM}}$ II SFM (GIBCO, 10902088) supplemented with 10\% fetal bovine serum (GIBCO, 10099141), and $1 \%$ Penicillin-Streptomycin (GIBCO, 15140163) at $27^{\circ} \mathrm{C}$.

\section{Plasmid construction}

DNA fragments encoding full-length sDscam isoforms or isoforms lacking the cytoplasmic domain were amplified by PCR using cDNA isolated from the scorpion Mesobuthus martensii 33. PCR products were cloned into the pEasy-blunt zero cloning vector (TransGen Biotech, CB501-01) and ligated into the pFastBacHTB-mCherry/EGFP expression vector with appropriate restriction enzyme sites to guarantee that the opening reading frame was correct and the tags of EGFP and mCherry were fused to the $\mathrm{C}$ terminal of target proteins. To generate pFastBacHTB-mCherry and pFastBacHTB-EGFP vectors, the full-length mCherry amplified from the pmCherry-N1 vector (a gift from Xinhua Feng, Zhejiang University) and EGFP amplified from the pEGFP-N1 vector (a gift from Naiming Zhou, Zhejiang University) were inserted into the pFastBacHTB vector (a gift from Xiaofeng Wu, Zhejiang University) using the KpnI (forward primer) and HindIII (reverse primer) restriction sites. Domain deletion and substitution recombinant pFastBacHTB-mCherry vectors were made by using overlapping PCR. Extracellular-Myc-tagged sDscam vectors inserting the c-Myc (EQKLISEEDL) tag after the FNIII3 domain of sDscam were also generated by using overlapping PCR. The single mutation, double mutations and the mutations between close sDscam pairs were generated by site directed mutagenesis (Quikchange method). To obtain the pFastBac1-Myc/HA vector, sequences encoding the Myc (EQKLISEEDL) / HA (YPYDVPDYA) peptide were synthesized and annealed to form duple strand and then cloned into the pFastBac1 vector (a gift from Chuanxi Zhang, Zhejiang University) using restriction sites SphI and KpnI. pFastBac1-Myc truncations were obtained by using overlapping PCR. All recombinant vectors were confirmed by DNA sequencing. Ig and FNIII domain were predicted using the PROSITE (https://prosite.expasy.org/). Signal peptides (SP) and transmembrane domains (TM) were 
predicted using SMART (http://smart.embl-heidelberg.de/). Primer sequences used for PCR amplifications will be provided upon request.

\section{Antibody generation}

Mouse monoclonal antibody against M. martensii sDscamß6V2 (amino acids M1-L198) was generated by the Huabio. $\beta 6 \mathrm{~V} 2$ antigen was cloned into pET-28a and transformed into Rosetta (DE3) E. coli, and purified using Ni-NTA beads (Smart-lifesciences) according to standard protocol.

\section{Recombinant baculovirus production}

Baculoviruses were obtained according to the manufacturer's instructions of Bac-to-Bac Baculovirus Expression System (GIBCO, 10359016). Briefly, to generate a recombinant bacmid, the pFastBac plasmid was transformed into DH10Bac competent cells (Biomed, $\mathrm{BC} 112$ ) and blue-white screening was used to pick the white colonies and recombinant bacmid DNA was analyzed by PCR. Recombinant bacmid was transfected into Sf9 cells using Lipofectamine 3000 Reagent (Invitrogen, L3000015). Cells were incubated at $27^{\circ} \mathrm{C}$ until see signs of viral infection. Then we harvested the virus from the cell culture medium to get P1 baculovirus. P1 viruses were added to $\mathrm{Sf9}$ cells grown in the 6-well plates, after $72 \mathrm{~h}$ of incubation at $27^{\circ} \mathrm{C}$, the cells were centrifuged to obtain the supernatant as P2 viruses. All baculoviruses should be stored at $4^{\circ} \mathrm{C}$.

\section{Cell aggregation assays}

Cell aggregation assay was performed as previously reported with little modification ${ }^{34}$. Sf9 cells grown in each well in 6-well plates were infected with P2 viral of target proteins tagged with mCherry or EGFP $10 \sim 30 \mathrm{ul}$ and incubated at $27^{\circ} \mathrm{C}$ for 3 days. Cells were suspended and transferred to the $2 \mathrm{ml}$ tube, centrifuged at 1,000 rpm for $5 \mathrm{~min}$. The supernatants were discarded, and the cell pellets were resuspended with the $1 \mathrm{ml} 1 \times \operatorname{HCMF}(1: 10$, Leagene Biotechnology, CC0073) gently and centrifuged at 1,000rpm for 5 min again. Cells were then resuspended with $1 \mathrm{ml} 1 \times \mathrm{HCMF}$ gently. For cell aggregation assay, 400ul cell suspension was transferred into 
each well in 6-well plates containing $2 \mathrm{ml} 1 \times \mathrm{HCMF}$ per well. For binding specificity assay, 200ul cell suspension of each sample was transferred into each well. The 6-well plates used in the cell aggregation assay firstly was added the $1 \%$ BSA in $1 \times \operatorname{HBSS}(1: 10$, Gibco, 14185052) at $4{ }^{\circ} \mathrm{C}$ overnight, then washed once with D-PBS and $2 \mathrm{ml} 1 \times \mathrm{HCMF}$ was added to each well. Cell suspension in 6-well plates was incubated at $27^{\circ} \mathrm{C}$ with gyratory shaker (IKA KS260) at 60rpm for $30 \mathrm{~min}$. Finally, images were captured using the Nikon Ti-S inverted fluorescence microscope.

\section{Binding specificity assay for cells expressing single or multiple sDscam isoform(s)}

Differentially tagged sDscam isoforms were infected into Sf9 cells as described above. We observed that sDscam's surface expression is different among sDscam $\alpha$ or sDscam $\beta$ isoforms in the coexpression experiments. Thus, sDscam $\alpha$-sDscam $\alpha$ or sDscam $\alpha$-sDscam $\beta$ were used in appropriate ratio roughly guaranteed the approximate equal surface expression. Images were captured using the Nikon Ti-S inverted fluorescence microscope, and the aggregates containing red cells only, green cells only, and both red and green cells (Red-Green) were observed and counted for analysis of binding specificity.

\section{Quantification of the size of cell aggregates using matlab}

The images of cells from three independent aggregation experiments were used to quantify the relative size of cell aggregates generated by each sDscam isoform. The images were first converted to black and white formats with $2160 \times 2560$ pixels. Objects with 1000 or fewer pixels were categorized as small ( $<10$ cells), objects between 1000 pixels and 6000 pixels were categorized as medium (10 to 80 cells), and objects larger than 6000 pixels were categorized as large ( $>80$ cells). The number of aggregates of each size category was then counted for analysis.

\section{Immunostaining}

Sf9 cells were seeded onto the coverslips (WHB Scientific) coated with 1mM poly-L-lysine (Sigma, P6282) in 6-well plates, and were infected by the viruses from the P2 stocks of sDscam $\alpha 14 \triangle$ cyto, sDscam $\beta 4 \mathrm{v} 3 \triangle$ cyto and sDscam $\alpha 14 \triangle$ cyto $\triangle \operatorname{Ig} 1$, these sDscam proteins 
inserted c-Myc tag between the FNIII3 and TM domain as well as carried a mCherry tag at the C terminus. after $72 \mathrm{hr}$, cells were fixed with 4\% Paraformaldehyde Fix Solution (PFA, Sangon Biotech, E672002-0500) for 20min at room temperature, then these non-permeabilized cells were washed three times with D-PBS (Sangon Biotech, E607009). Cells were blocked with 5\% BSA in PBS and incubated with anti-Myc tag monoclonal antibody (1:400, Earthox, E02205001) overnight at $4{ }^{\circ} \mathrm{C}$. Subsequently, cells were washed three times with PBS, incubated with goat Anti-Mouse IgG (H+L) Dylight488 (1:500, Earthox, E032210-01) diluted in 5\% BSA of D-PBS for $1 \sim 2 \mathrm{~h}$ at room temperature and then washed three times with D-PBS. Finally, cell was stained by Hoechst ( $2 \mu \mathrm{g} / \mathrm{ml}$, invitrogen, Hoechst 33342) for 15 30 min for nucleus staining and imaging with a laser scanning confocal microscope LSM800 (Carl Zeiss).

\section{Co-immunoprecipitation and western blot analysis}

Sf9 cells grown in 6-well plates were infected with viral and incubated for 3 days at $27^{\circ} \mathrm{C}$. Cells were lysed in the IP lysis buffer (Thermo Fisher Scientific, 87787). The cells were incubated in lysis buffer for $30 \mathrm{~min}$ at $4^{\circ} \mathrm{C}$. The supernatant was collected by centrifugation at $13,000 \mathrm{~g}$ for $20 \mathrm{~min}$ at $4^{\circ} \mathrm{C}$. Subsequently, cellular extracts were incubated with appropriate antibodies at $4^{\circ} \mathrm{C}$ overnight, followed by incubating with pre-washed protein A/G magnetic beads (Thermo Fisher Scientific, 88802 ) for $3 \mathrm{~h}$ at $4^{\circ} \mathrm{C}$. The beads were collected and washed, then boiled with SDS sample buffer (Sangon Biotech, C508320-0001) for $10 \mathrm{~min}$. The beads were separated and then the supernatant was saved for western blotting according to the standard methods. In brief, proteins were electrophoresed in Tris-Glycine gel (Sangon Biotech, C651101-0001) and transferred to the PVDF membranes (Millipore, IPVH00010). Membranes were incubated with antibodies as described and then performed protein signal detection. For multimer detection of sDscam, target proteins in Sf9 cell and tissues were extracted using the RIPA lysis buffer (strong) (Cowin Biosciences, CW2333S) freshly supplemented with 1\% protease inhibitor cocktail. The sample was treated with nonreducing sample buffer (Sangon Biotech,C516031) without boiling then directly centrifuged at $13,000 \mathrm{rpm}$ for $20 \mathrm{~min}$ at $4{ }^{\circ} \mathrm{C}$. The proteins were electrophoresed in Tris-Glycine PAGE gel (Sangon Biotech, C651104-0001). Then the western blotting was performed as described above. The anti-Mma $\beta 6 \mathrm{~V} 2$ monoclonal antibody (produced by Huabio, 
Hangzhou) raised against the Ig1 domain of Mma $\beta 6 \mathrm{~V} 2$ was used by tissue western blotting.

The antibodies used in IP: anti-HA Tag rabbit polyclonal antibody (1:50, Earthox, E02218001), HA-Tag (C29F4) rabbit mAb (1:50, Cell Signaling Technology, 3724S), anti-GFP rabbit polyclonal antibody (1:50, Earthox, E022200-01). The primary antibodies used in Western blotting: anti-HA Tag monoclonal antibody (1:5000, Earthox, E022010-01), anti-Myc Tag antibody (1:5000, Earthox, E022050-01), anti-mCherry Tag antibody (1:5000, Earthox, E022110-01), anti-GFP Tag mouse monoclonal antibody (1:5000, Earthox, E022030-01), AntisDscam $\beta 6$ V2 Monoclonal Antibody (1:1000). The secondary antibodies used in Western blotting: HRP AffiniPure goat anti-Mouse IgG (1:8000, Earthox, E030110-01).

\section{Homology modeling and protein-protein docking}

Ig1 homodimeric models of sDscam were built with swiss-model (https://www.swissmodel) using Dscam Ig7 as a temple ${ }^{39,40,46}$. Macromolecular interface was furthermore explored using PDBePISA (https://www.ebi.ac.uk/msd-srv/prot int/cgi-bin/piserver) ${ }^{47}$, the results show some buried residues and their potential interaction (hydrogen/disulphide bond, salt bridge or covalent link), single and double complementary mutations of these candidate residues were conducted upon this. Specificity-determining residues of six sDscam $\alpha$ pairs were screened also based on homology modeling. Similarly, TM domain of sDscam $\beta 6 \mathrm{v} 2$ was generated by swissmodel, and all these structure figures were prepared with VMD package (https://www.ks.uiuc.edu/Research/vmd/). In addition, Ig3-FNIII3 domain of sDscam $\beta 6$ v2 was predicted as monomer by swiss-model and then was used for homologous dimer docking by ZDOCK server (http://zdock.umassmed.edu/m-zdock/). Finally, the dimer interface were visualized with the PyMOL package (www.pymol.com).

\section{Sequence alignments and heatmap analysis}

Multi-sequence alignments of Ig1 and Ig1-2 domains of the sDscam were carried out with Clustal Omega (https://www.ebi.ac.uk/Tools/msa/clustalo/) and Align (https://www.uniprot.org/align/). Then the sequence similarity heatmap was made by $\mathrm{R}$ language. 


\section{Statistics}

Statistical significance was calculated by using IBM SPASS Statistics V22.0 (Mann-Whitney U-test) to determine significant difference of cell aggregation size between TM domains shuffled isoforms, as well as $\beta 6 \mathrm{v} 2 \mathrm{FL}-\mathrm{Cherry}$ and $\beta 6 \mathrm{v} 2 \Delta$ cyto-Cherry isoforms. The number of independent experiment of duplicates, the statistical significance, and the statistical test are indicated in each figure or figure legend where quantification is reported.

\section{Author contributions}

YJ conceived of this project. FZ, GC, CD, GL, HL, and BX designed and performed the experiments; FZ, GC, CD, GL, and HL conducted cell aggregation assays and binding specificity assay; FZ, GC, CD and GL performed multimerization assay; GC conducted sDscam expression and antibody generation; GC, CD, GL, and FZ, performed co-immunoprecipitation and western blot analysis; FZ, SH and ZD performed homology modeling and protein-protein docking; YJ, FZ, BX, FS, YD and XY analyzed the data; YJ and FZ wrote the manuscript; all authors discussed the results and commented on the manuscript.

\section{Acknowledgements}

This work was supported by research grants from the National Natural Science Foundation of China $(31630089,31430050,91740104)$. 


\section{References}

1. Zipursky SL, Grueber WB. The molecular basis of self-avoidance. Annu Rev Neurosci 36, 547-568 (2013).

2. Mountoufaris G, Canzio D, Nwakeze CL, Chen WV, Maniatis T. Writing, Reading, and Translating the Clustered Protocadherin Cell Surface Recognition Code for Neural Circuit Assembly. Annu Rev Cell Dev Biol 34, 471-493 (2018).

3. Zipursky SL, Sanes JR. Chemoaffinity revisited: dscams, protocadherins, and neural circuit assembly. Cell 143, 343-353 (2010).

4. Wojtowicz WM, Flanagan JJ, Millard SS, Zipursky SL, Clemens JC. Alternative splicing of Drosophila Dscam generates axon guidance receptors that exhibit isoformspecific homophilic binding. Cell 118, 619-633 (2004).

5. Wojtowicz WM, Wu W, Andre I, Qian B, Baker D, Zipursky SL. A vast repertoire of Dscam binding specificities arises from modular interactions of variable Ig domains. Cell 130, 1134-1145 (2007).

6. Hattori D, Millard SS, Wojtowicz WM, Zipursky SL. Dscam-mediated cell recognition regulates neural circuit formation. Annu Rev Cell Dev Biol 24, 597-620 (2008).

7. Miura SK, Martins A, Zhang KX, Graveley BR, Zipursky SL. Probabilistic splicing of Dscam1 establishes identity at the level of single neurons. Cell 155, 1166-1177 (2013).

8. Neves G, Zucker J, Daly M, Chess A. Stochastic yet biased expression of multiple Dscam splice variants by individual cells. Nat Genet 36, 240-246 (2004).

9. Schmucker D, et al. Drosophila Dscam is an axon guidance receptor exhibiting extraordinary molecular diversity. Cell 101, 671-684 (2000).

10. Sun W, et al. Ultra-deep profiling of alternatively spliced Drosophila Dscam isoforms by circularization-assisted multi-segment sequencing. EMBO J 32, 2029-2038 (2013).

11. Hattori D, et al. Robust discrimination between self and non-self neurites requires thousands of Dscam1 isoforms. Nature 461, 644-648 (2009).

12. Hattori D, Demir E, Kim HW, Viragh E, Zipursky SL, Dickson BJ. Dscam diversity is essential for neuronal wiring and self-recognition. Nature 449, 223-227 (2007).

13. He H, et al. Cell-intrinsic requirement of Dscam1 isoform diversity for axon collateral formation. Science 344, 1182-1186 (2014).

14. Jin Y, Li H. Revisiting Dscam diversity: lessons from clustered protocadherins. Cell Mol Life Sci 76, 667-680 (2019).

15. Schmucker D, Chen B. Dscam and DSCAM: complex genes in simple animals, complex animals yet simple genes. Genes Dev 23, 147-156 (2009).

16. Yagi T. Molecular codes for neuronal individuality and cell assembly in the brain. Front Mol Neurosci 5, 45 (2012).

17. Wu Q, Maniatis T. A striking organization of a large family of human neural cadherinlike cell adhesion genes. Cell 97, 779-790 (1999).

18. Wu Q, et al. Comparative DNA sequence analysis of mouse and human protocadherin gene clusters. Genome Res 11, 389-404 (2001).

19. Ribich S, Tasic B, Maniatis T. Identification of long-range regulatory elements in the protocadherin-alpha gene cluster. Proc Natl Acad Sci U S A 103, 19719-19724 (2006).

20. Tasic B, et al. Promoter choice determines splice site selection in protocadherin alpha 
and gamma pre-mRNA splicing. Mol Cell 10, 21-33 (2002).

21. Wang X, Su H, Bradley A. Molecular mechanisms governing Pcdh-gamma gene expression: evidence for a multiple promoter and cis-alternative splicing model. Genes Dev 16, 1890-1905 (2002).

22. Schreiner D, Weiner JA. Combinatorial homophilic interaction between gammaprotocadherin multimers greatly expands the molecular diversity of cell adhesion. Proc Natl Acad Sci U S A 107, 14893-14898 (2010).

23. Thu CA, et al. Single-cell identity generated by combinatorial homophilic interactions between alpha, beta, and gamma protocadherins. Cell 158, 1045-1059 (2014).

24. Brasch J, et al. Visualization of clustered protocadherin neuronal self-recognition complexes. Nature 569, 280-283 (2019).

25. Goodman KM, et al. Protocadherin cis-dimer architecture and recognition unit diversity. Proc Natl Acad Sci U S A 114, E9829-E9837 (2017).

26. Goodman KM, et al. Structural Basis of Diverse Homophilic Recognition by Clustered alpha- and beta-Protocadherins. Neuron 90, 709-723 (2016).

27. Nicoludis JM, Lau SY, Scharfe CP, Marks DS, Weihofen WA, Gaudet R. Structure and Sequence Analyses of Clustered Protocadherins Reveal Antiparallel Interactions that Mediate Homophilic Specificity. Structure 23, 2087-2098 (2015).

28. Nicoludis JM, Vogt BE, Green AG, Scharfe CP, Marks DS, Gaudet R. Antiparallel protocadherin homodimers use distinct affinity- and specificity-mediating regions in cadherin repeats 1-4. Elife 5, (2016).

29. Rubinstein R, et al. Molecular logic of neuronal self-recognition through protocadherin domain interactions. Cell 163, 629-642 (2015).

30. Lefebvre JL, Kostadinov D, Chen WV, Maniatis T, Sanes JR. Protocadherins mediate dendritic self-avoidance in the mammalian nervous system. Nature 488, 517-521 (2012).

31. Mountoufaris G, et al. Multicluster Pcdh diversity is required for mouse olfactory neural circuit assembly. Science 356, 411-414 (2017).

32. Cao $\mathrm{G}$, et al. A chelicerate-specific burst of nonclassical Dscam diversity. BMC Genomics 19, 66 (2018).

33. Yue Y, et al. A large family of Dscam genes with tandemly arrayed 5' cassettes in Chelicerata. Nat Commun 7, 11252 (2016).

34. Sasakura $\mathrm{H}$, et al. Maintenance of neuronal positions in organized ganglia by SAX-7, a Caenorhabditis elegans homologue of L1. EMBO J 24, 1477-1488 (2005).

35. Bonn S, Seeburg PH, Schwarz MK. Combinatorial expression of alpha- and gammaprotocadherins alters their presenilin-dependent processing. Mol Cell Biol 27, 41214132 (2007).

36. Murata Y, Hamada S, Morishita H, Mutoh T, Yagi T. Interaction with protocadheringamma regulates the cell surface expression of protocadherin-alpha. J Biol Chem 279, 49508-49516 (2004).

37. Li SA, Cheng L, Yu Y, Chen Q. Structural basis of Dscam1 homodimerization: Insights into context constraint for protein recognition. Sci Adv 2, e1501118 (2016).

38. Sawaya MR, et al. A double S shape provides the structural basis for the extraordinary binding specificity of Dscam isoforms. Cell 134, 1007-1018 (2008).

39. Guex N, Peitsch MC, Schwede T. Automated comparative protein structure modeling 
with SWISS-MODEL and Swiss-PdbViewer: a historical perspective. Electrophoresis 30 Suppl 1, S162-173 (2009).

40. Waterhouse A, et al. SWISS-MODEL: homology modelling of protein structures and complexes. Nucleic Acids Res 46, W296-W303 (2018).

41. Pierce BG, Wiehe K, Hwang H, Kim BH, Vreven T, Weng Z. ZDOCK server: interactive docking prediction of protein-protein complexes and symmetric multimers. Bioinformatics 30, 1771-1773 (2014).

42. Dong Y, Cirimotich CM, Pike A, Chandra R, Dimopoulos G. Anopheles NF-kappaBregulated splicing factors direct pathogen-specific repertoires of the hypervariable pattern recognition receptor AgDscam. Cell Host Microbe 12, 521-530 (2012).

43. Li D, et al. Alternatively spliced down syndrome cell adhesion molecule (Dscam) controls innate immunity in crab. J Biol Chem 294, 16440-16450 (2019).

44. Watson FL, et al. Extensive diversity of Ig-superfamily proteins in the immune system of insects. Science 309, 1874-1878 (2005).

45. Chen WV, et al. Pcdhalphac2 is required for axonal tiling and assembly of serotonergic circuitries in mice. Science 356, 406-411 (2017).

46. Bienert S, et al. The SWISS-MODEL Repository-new features and functionality. Nucleic Acids Res 45, D313-D319 (2017).

47. Krissinel E, Henrick K. Inference of macromolecular assemblies from crystalline state. J Mol Biol 372, 774-797 (2007). 
$\mathbf{a}$

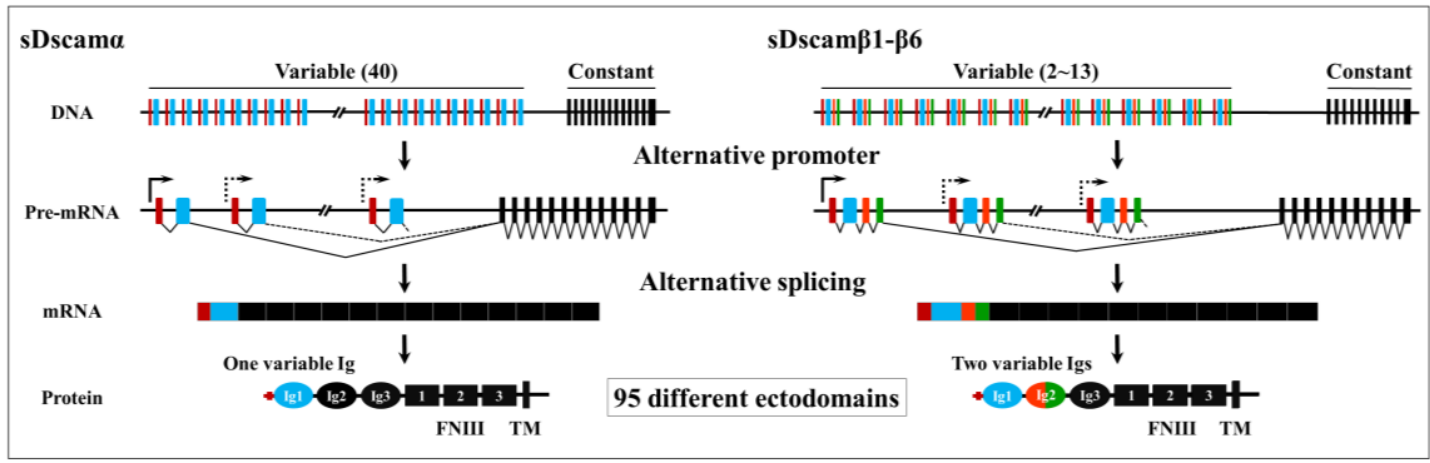

b

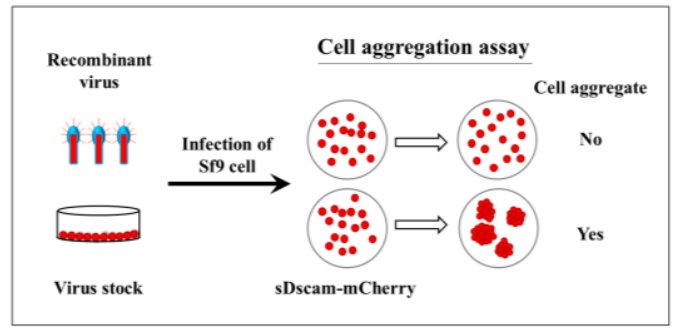

c

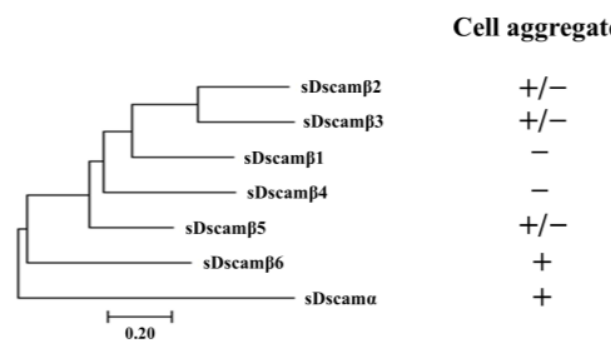

d
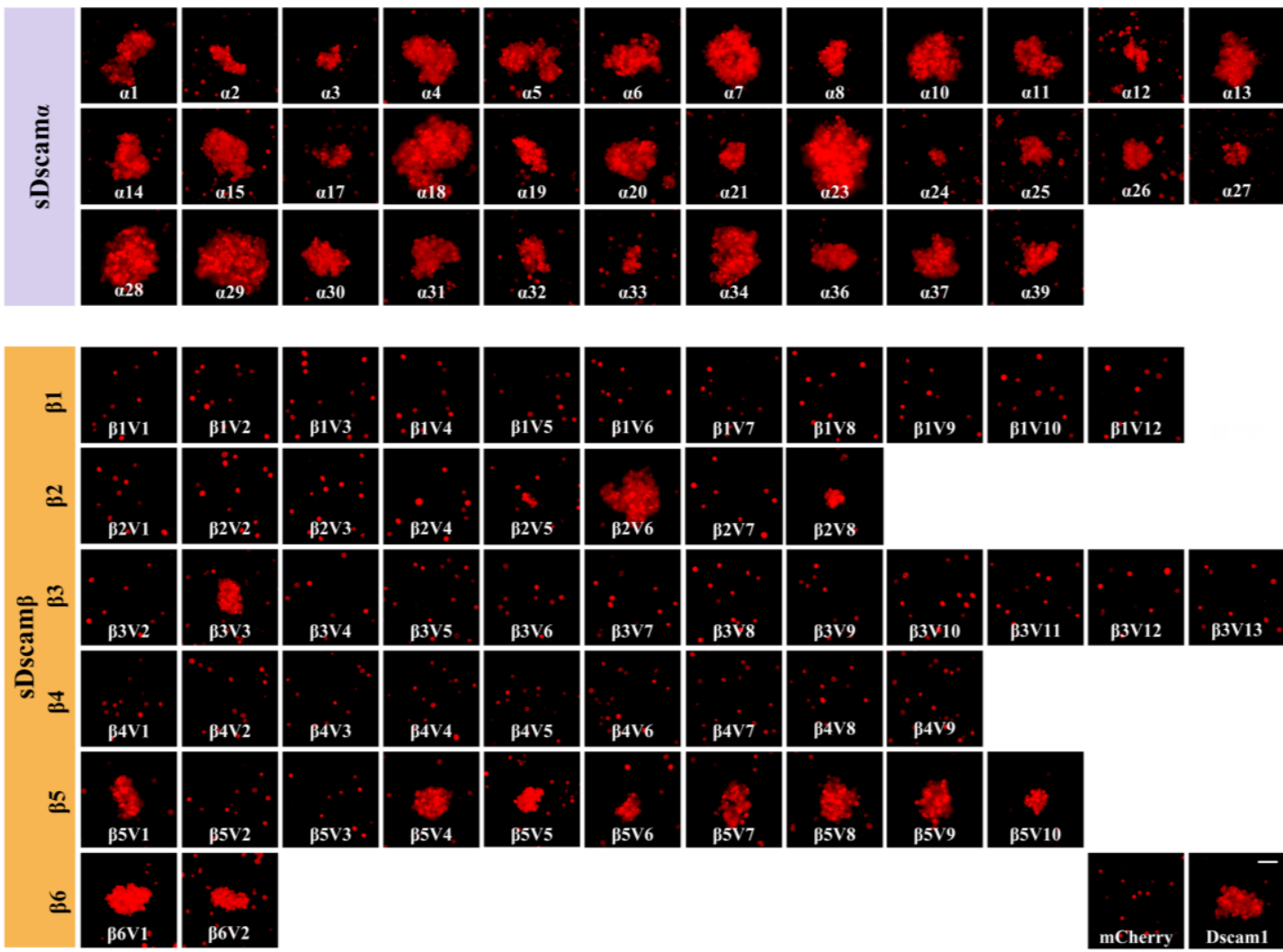

Fig. 1 Cluster-wide analysis of sdscam-mediated homophilic binding in M. martensii.

(a) Overview of the M. martensii sDscam gene clusters. Variable exons (coloured) are joined via $c i s$-splicing to the constant exons (black) in sDscam $\alpha$ (left) and sDscam $\beta 1-\beta 6$ (right) subfamilies. Each variable cassette of sDscam $\alpha$ encodes Ig1 domain, while that of sDscam $\beta$ 
encodes Ig1-2 domains. The constant exons of sDscam $\alpha$ and sDscam $\beta$ encode the $\operatorname{Ig} 2-3$ or Ig3 domains, FNIII1-3 domains, the transmembrane and cytoplasmic domains.

(b) Schematic diagram of the cell aggregation assay. mCherry-tagged sDscam proteins were expressed in Sf9 cells for assaying their ability to form cell aggregates. As shown in the diagram, cells expressing some sDscam-mCherry alone did not aggregate as negative control mCherry, while strong cell aggregates were observed with cells expressing other sDscammCherry as positive control Dscam1-mCherry.

(c) The summary of results for homophilic binding properties. An evolutionary relationship among distinct sDscam subfamilies is shown on the left.

(d) The outcome of cell aggregation of 85 sDscam isoforms when assaying individually. mCherry and fly Dscam1 isoform were expressed as negative and positive control. See also Supplementary Fig. 1b. Scale bar, $100 \mu \mathrm{m}$. 
$\mathbf{a}$

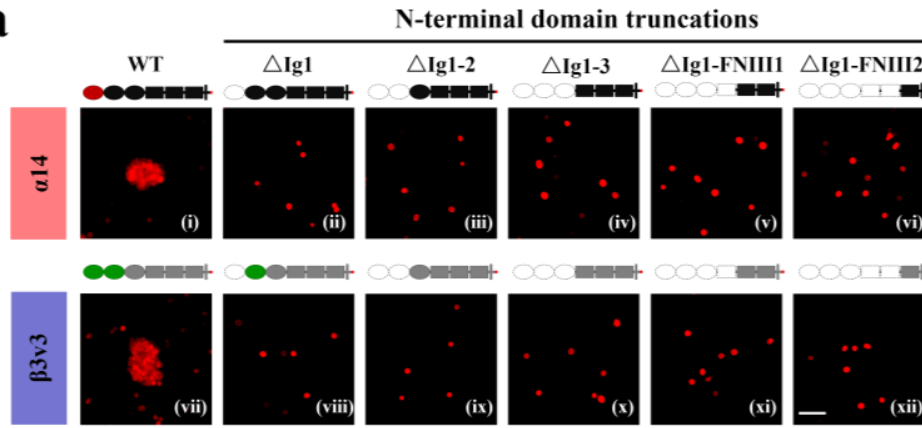

c

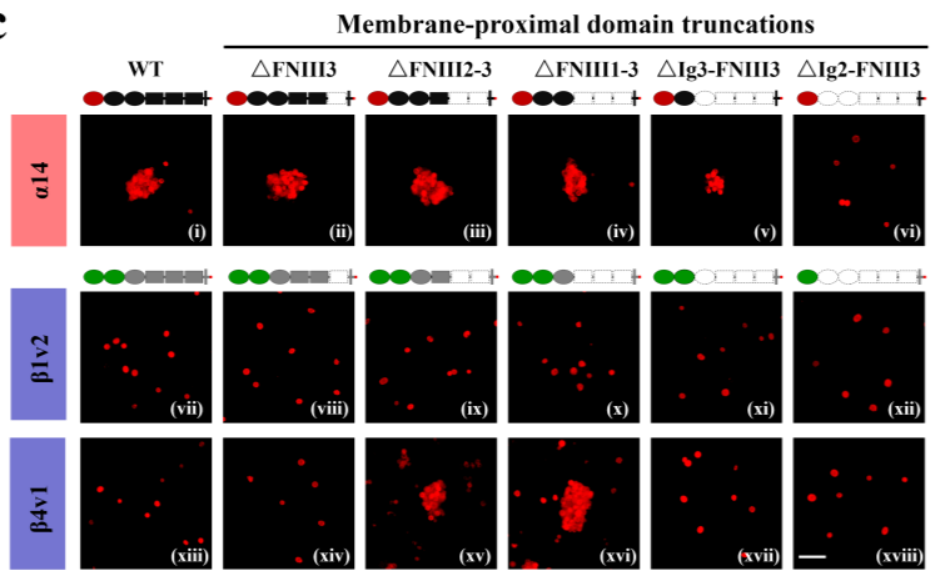

b

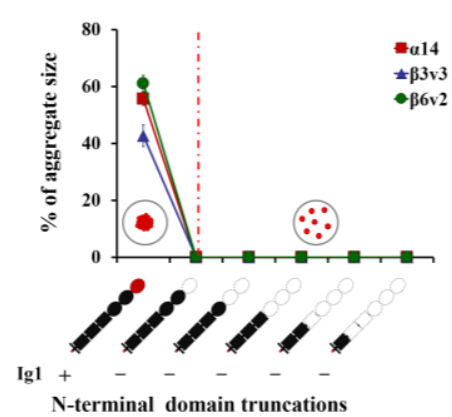

d

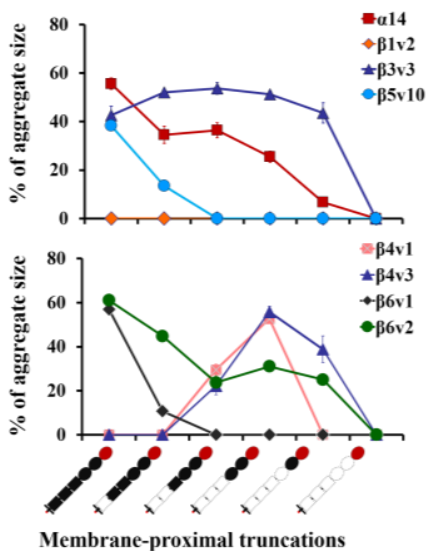

e

sDscam domain shuffling

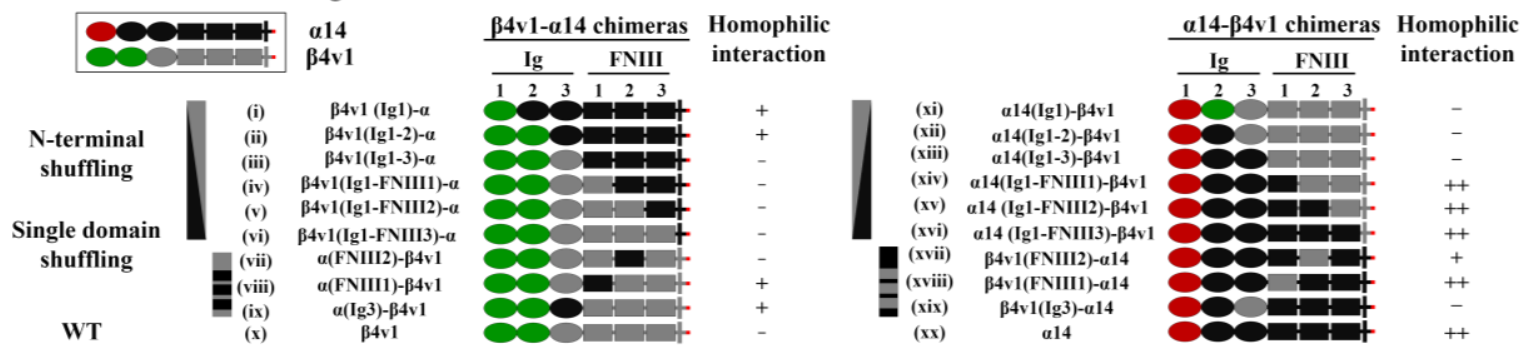

f

N-terminal domains-shuffled chimeras

Single domain-shuffled chimeras

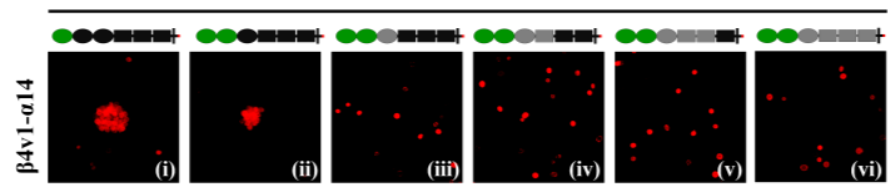

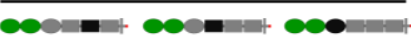
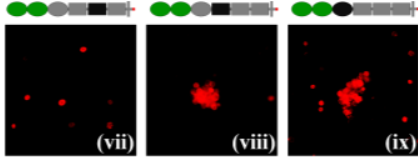

B4v1-WT

$\infty)^{\infty}$

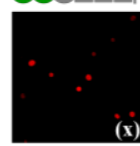

$\mathrm{N}$-terminal domains-shuffled chimeras

Single domain-shuffled chimeras
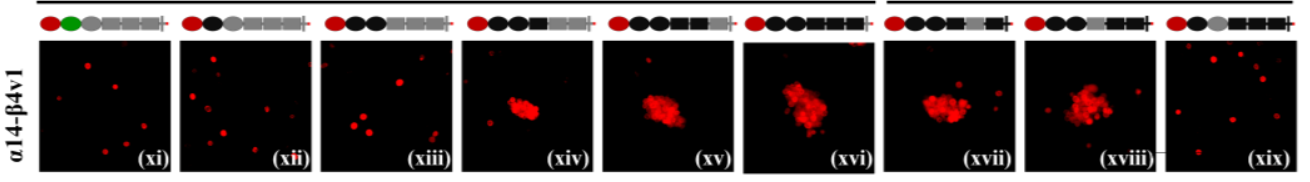

a14-WT

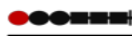

g

sDscam a39-ק3 TM domain shuffling
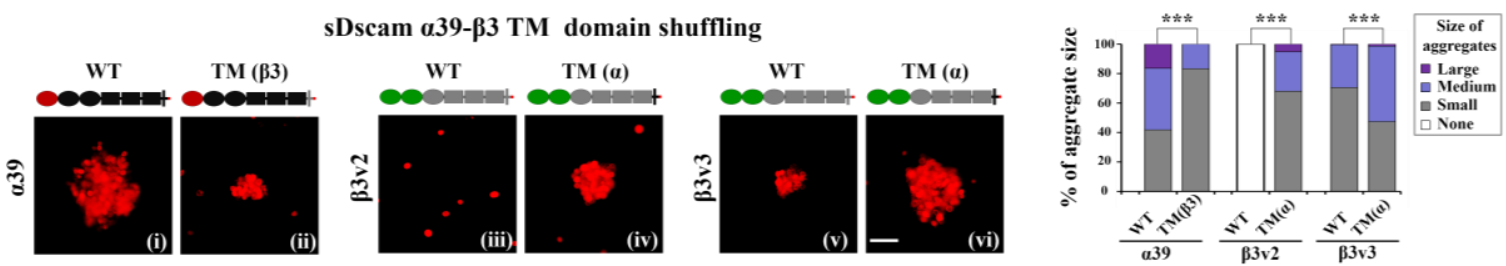
Fig. 2 Homophilic trans-binding is associated with variable and constant domains of sDscam.

(a) A series of N-terminal truncations of the extracellular domain of sDscam fused with mCherry were examined for cell aggregation assay. Three independent assays were performed in sDscam $\alpha 14$, sDscam $\beta 3 \mathrm{v} 3$ and $\mathrm{sDscam} \beta 6 \mathrm{v} 2$, respectively. All of the sDscam truncations lacking the N-terminal Ig1 domain failed to form cell aggregate. See also Supplementary Fig. 2a.

(b) The ratio change of cell aggregate size (medium and large) of sDscam N-terminal truncations. Results were obtained from three independent experiments and expressed as mean \pm SEM.

(c) The first two N-terminal domains are required for trans homophilic binding. Eight sDscam isoforms including $1 \mathrm{sDscam} \alpha$ and $7 \mathrm{sDscam} \beta \mathrm{s}$ isoforms were deleted starting with the membrane-proximal FNIII3 domain, and these truncations were tested for cell aggregation. These data showed that all of the truncated constructs containing only one Ig1 domain failed to form cell aggregates, and the smallest truncations that exhibited binding ability contain $\mathrm{N}$ terminal Ig1-2 domains. Supplementary Fig. 2a.

(d) Homophilic trans-binding is associated with constant extracellular domains of sDscam. The graph showed that cell aggregation size (medium and large) of many sDscam truncations changed obviously, except sDscam $\beta 1 v 1$. See also Supplementary Fig. 2a. Results were obtained from three independent experiments and expressed as mean $\pm \mathrm{SEM}$.

(e) Schematic diagrams for domain shuffling mutants used in the experiments, along with a summary of outcomes from homophilic interaction assays. Extracellular domain of sDscam $\alpha 14$ (red and black) were replaced with the corresponding domains of sDscam $\beta 4 \mathrm{v} 1$ (green and gray), or vice versa. All of the chimeras bearing the Ig3-FNIII1 domain from sDscama14 (black) could affect cell aggregation. See also Supplementary Fig. 2b, c.

(f) Cell adhesion images were presented corresponding to the shuffled chimeras shown in Fig. $2 \mathrm{e}(\mathrm{i}-\mathrm{xx})$. These results indicate that homophilic binding is regulated by constant extracellular domains of sDscam.

(g) The TM domain is associated with homophilic binding. TM domain shuffling experiments between sDscam $\alpha 39$ and sDscam $\beta 3 \mathrm{v} 2 / \mathrm{v} 3$ were performed. Right histograms show the 
proportion of each category of the quantification of the cell aggregation corresponding to the shuffled chimeras (panels i-vi). Results were obtained from three independent experiments and expressed as mean. Mann-Whitney U-test was performed to determine significance. $* * * p<$ 0.001. Scale bar, $100 \mu \mathrm{m}$. 
bioRxiv preprint doi: https://doi org/10.1101/2019.12 15 877159; this version posted December 16, 2019. The copyright holder for this preprint (which was not certified by peer review) is the author/funder, who has granted bioRxiv a license to display the preprint in perpetuity. It is made available under aCC-BY-NC-ND 4.0 International license.

a

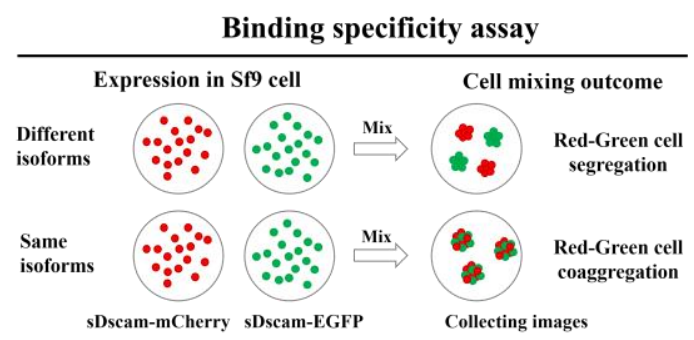

b

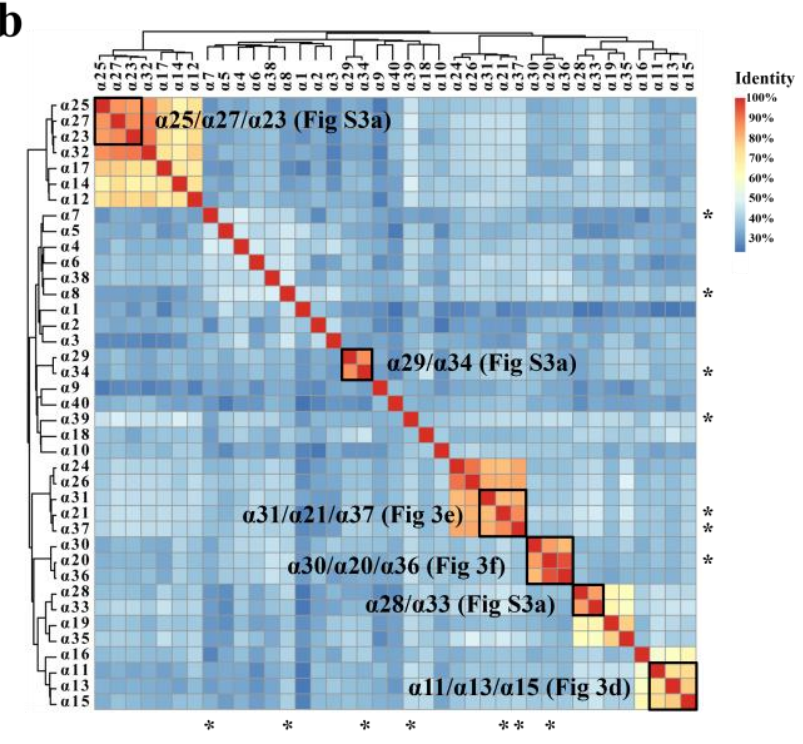

c

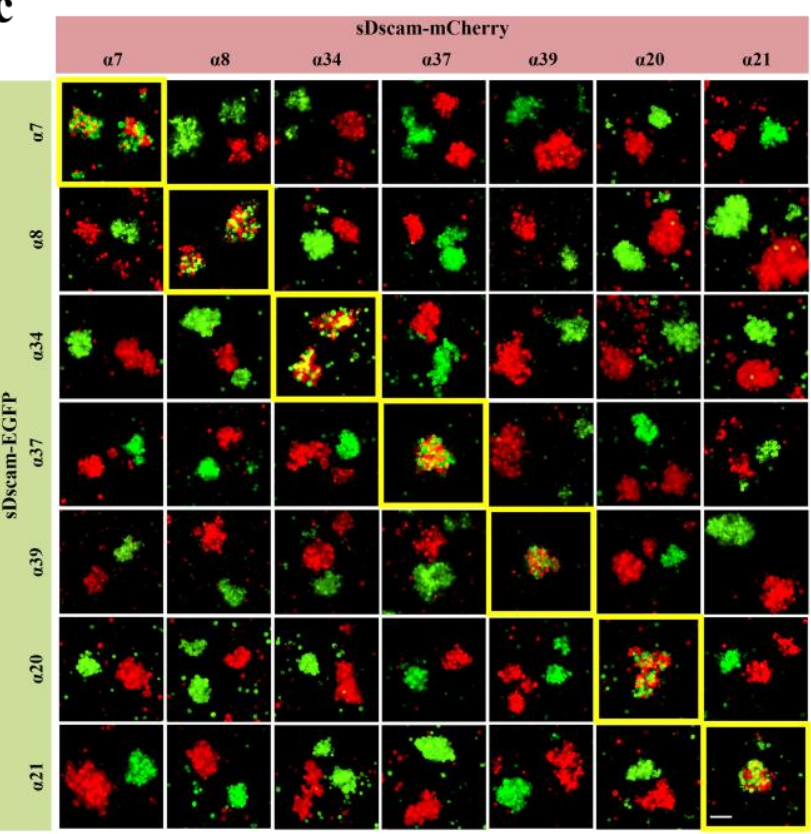

d

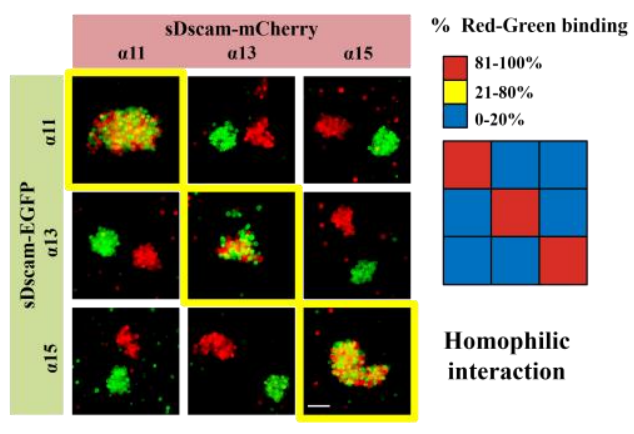

e

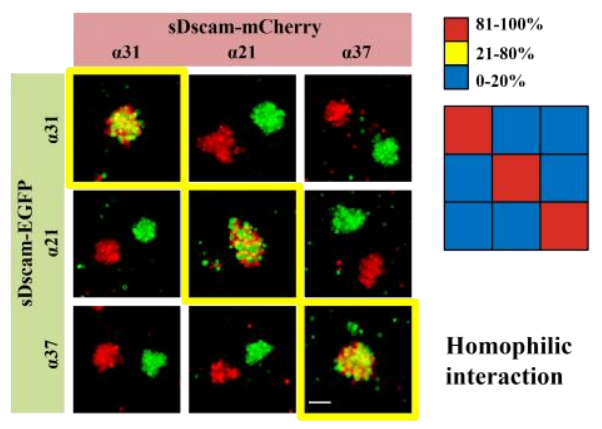

f

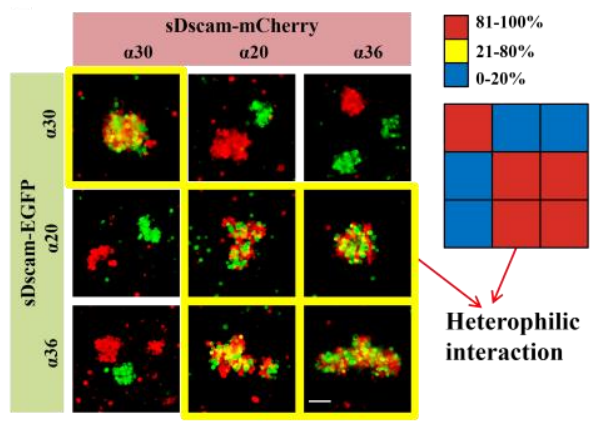

g

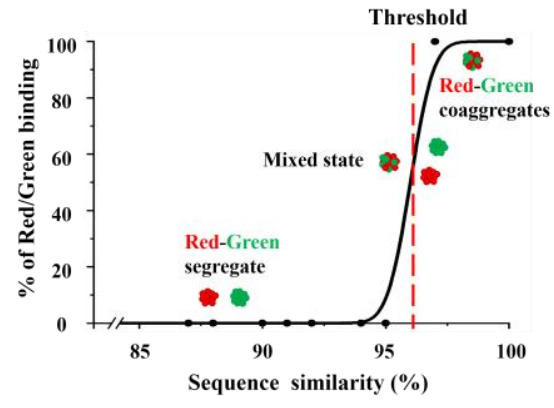

Fig. 3 sDscam isoforms engaged in highly specific homophilic interactions.

(a) Schematic diagram of the binding specificity assay. Cells expressing mCherry- or EGFPtagged sDscam isoforms were mixed and assayed for homophilic or heterophilic binding. The outcome of cell aggregation included red-green cell segregation and red-green cell 
coaggregation.

(b) Heat map of pairwise amino acid sequence identities of the Ig1 domains of sDscam $\alpha$ isoforms and their evolutionary relationship. Subsets of the isoforms marked by "**” and within the boxed region were assayed in Fig. 3c-f. See also Supplementary Fig. 3a.

(c-e) sDscam $\alpha$ isoforms with sequence identity for nonself pairs ranging from $50 \%$ to $94 \%$ in their Ig domains display strict trans- homophilic specificity. Pairwise combinations within representative sDscamas were assayed for their binding specificity. Scale bar, $100 \mu \mathrm{m}$.

(f) Pairwise combinations within sDscama20\&30\&36 pairs were assayed for their binding specificity. sDscama36 exhibited strong heterophilic binding to sDscama20, but did not to sDscama30.

(g) Sequence identity of the Ig1 domains correlated with their binding specificity. Comparative analysis indicates that the thresholds for homophilic and heterophilic binding is set by $\sim 96 \%$ sequence identity within their Ig1 domains between sDscam $\alpha$ pairs. Cell mixing outcome of different sDscam $\alpha$ pairs with sequence identity lower than threshold ( $96 \%)$ showed red-green segregation, while red-green aggregation was observed in sDscam $\alpha$ pairwise combinations with sequence identity higher than threshold. 
a

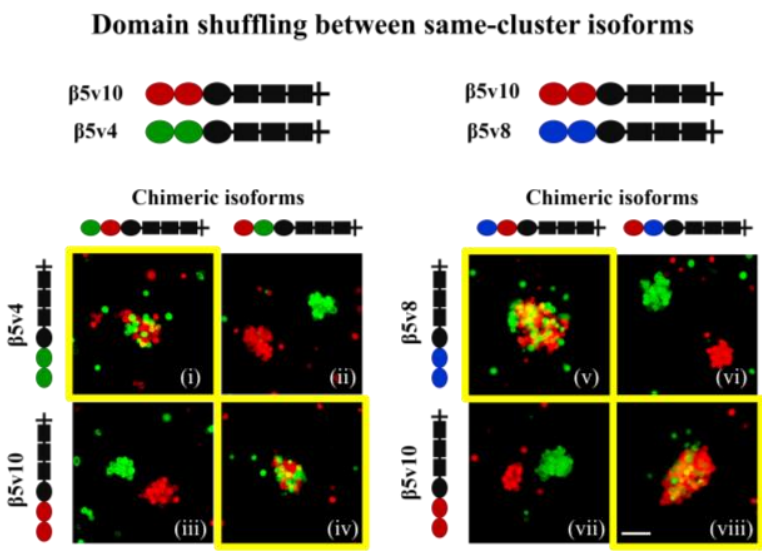

b

Between different-cluster isoforms

$\beta 5 \mathrm{v} 10 \mathrm{OOH}+\mathrm{On}+$

B6v1

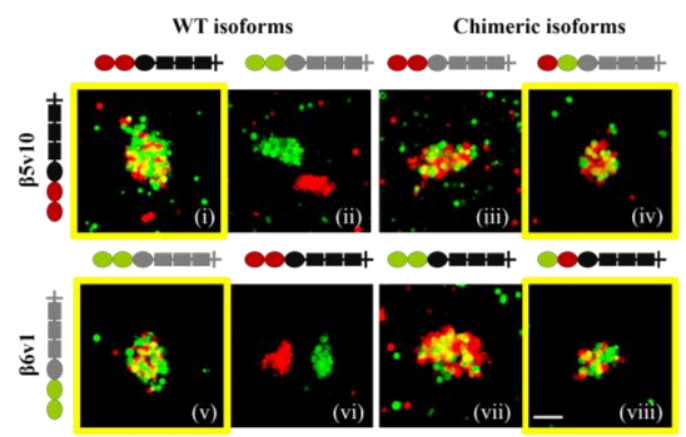

d

sDscam Ig1 determines trans-binding specificity

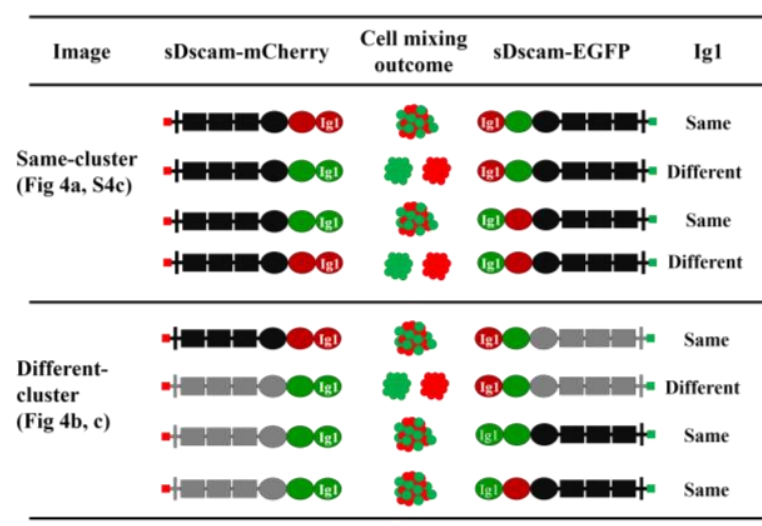

Fig. 4 sDscam trans-binding specificity is largely dependent on N-terminal Ig1 domain.

(a) Domain-shuffled chimeras of sDscam $\beta 5$ isoforms and their parental counterparts were assayed for binding specificity. Chimeras in which either the Ig1 or Ig2 domains were replaced with the corresponding domains of cluster-within isoforms swapped or not swapped transbinding specificity. See also Supplementary Fig. 4a, b.

(b) Swapped specificity was shown in sDscam $\beta 5 \mathrm{v} 10$ and sDscam $\beta 6 \mathrm{v} 1$ chimeras. These chimeras were replaced either the $\operatorname{Ig} 1-2$ or single $\operatorname{Ig} 1 / \operatorname{Ig} 2$ domains with the corresponding domains of different cluster isoforms. See also Supplementary Fig. 4a, b.

(c) Domain-shuffled chimeras between sDscam $\alpha$ and sDscam $\beta 3$ and their parental counterparts were assayed for binding specificity. See also Supplementary Fig. 4a, b.

(d) A summary of schematic of the domain-shuffled sDscam chimeras and their observed binding specificities. These data indicate that the presence of a single common Ig1 domain is 
bioRxiv preprint doi: https://doi.org/10.1101/2019.12.15.877159; this version posted December 16, 2019. The copyright holder for this preprint (which was not certified by peer review) is the author/funder, who has granted bioRxiv a license to display the preprint in perpetuity. It is made available under aCC-BY-NC-ND 4.0 International license.

essential and sufficient to confer co-aggregation between sDscam isoforms. Scale bar, $100 \mu \mathrm{m}$. 
bioRxiv preprint doi: https://doi.org/10.1101/2019.12.15.877159; this version posted December 16, 2019. The copyright holder for this preprint (which was not certified by peer review) is the author/funder, who has granted bioRxiv a license to display the preprint in perpetuity. It is made available under aCC-BY-NC-ND 4.0 International license.

a

Ig1 interface docking model

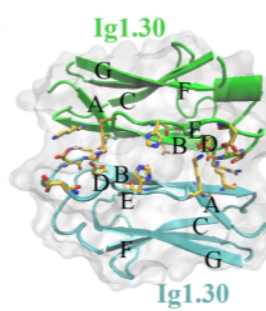

c

(i)

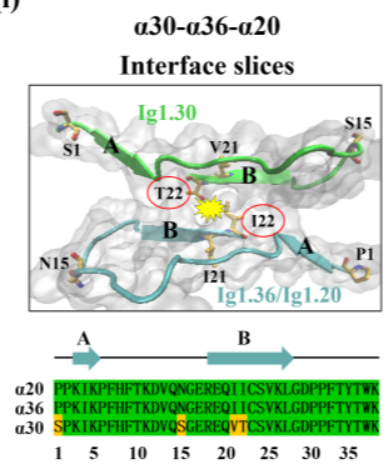

d

(i)

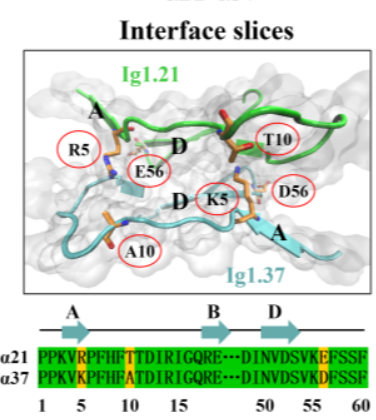

b
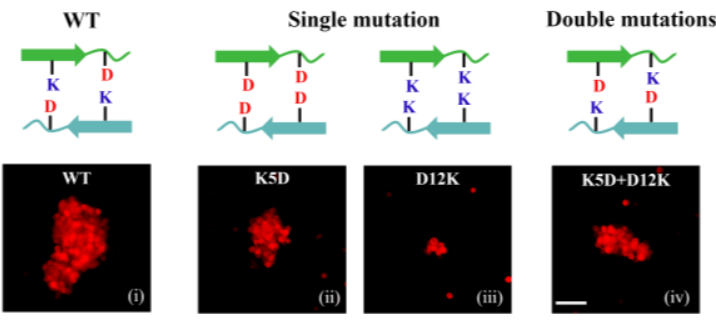

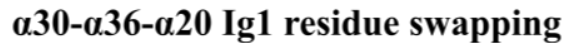

(ii)

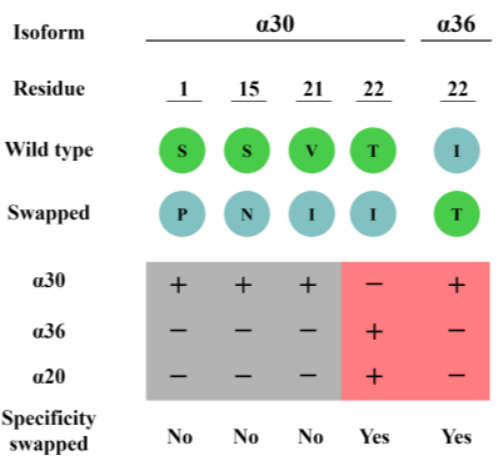

(iii)

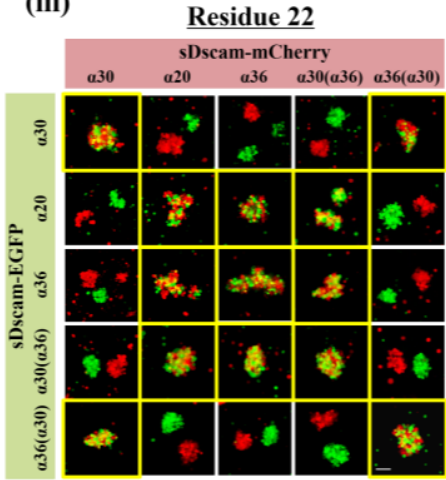

(ii)

\section{$\alpha 21-\alpha 37$ Ig1 residue swapping}

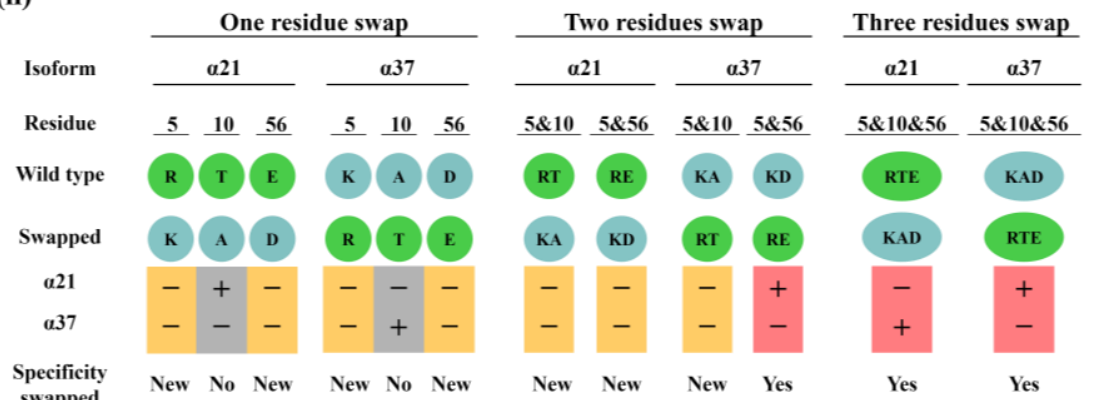

(iii)

\begin{tabular}{|c|c|c|c|c|c|c|c|c|c|c|c|c|c|c|}
\hline \multirow[t]{2}{*}{$\alpha 21-\alpha$} & \multirow{2}{*}{\multicolumn{4}{|c|}{$\underbrace{\text { Residue 5 }}_{\text {sDscam-mCherry }}$}} & \multicolumn{5}{|c|}{$\frac{\text { Residue } 5 \text { \& } 56}{\text { sDscam-mCherry }}$} & & \multicolumn{4}{|c|}{$\underbrace{\text { Residue } 5 \& 10 \text { \& } 56}_{\text {sDscam-mCherry }}$} \\
\hline & & & & & & $\alpha 21$ & $\begin{array}{l}\text { Dscam } \\
\text { a37 }\end{array}$ & $\begin{array}{l}\text { nCherry } \\
\alpha 21(a 37)\end{array}$ & $\alpha 37(a 21)$ & & $\alpha 21$ & Sscam & $\begin{array}{l}n C h e r r y \\
\alpha 21(a 37)\end{array}$ & $\alpha 37(\alpha 21)$ \\
\hline $\bar{ช}$ & & $\begin{array}{l}8 \\
: 8\end{array}$ & 9 & 88 & ฐ্ & \& & $\begin{array}{l}3 \\
8 \\
\end{array}$ & 48 & 9 & $\bar{g}$ & 年 & $\begin{array}{l}2 y: \\
8 \%\end{array}$ & 6 & 6. \\
\hline 产 & $\Leftrightarrow$ & 舟 & 8 & & $\widehat{\frac{1}{5}} \sqrt{3}$ & \pm & क & 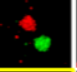 & $\Leftrightarrow$ & 둥 & 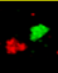 & 我 & $\phi$ & 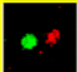 \\
\hline 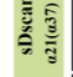 & 8 & $=8$ & \$ & 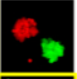 & 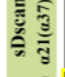 & $=6$ & 4. & 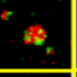 & set & 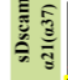 & 4 & \& & 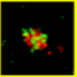 & 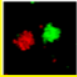 \\
\hline$\frac{\overline{3}}{3}$ & 8 & $8^{\circ}$ & 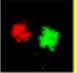 & es & $\frac{\widehat{3}}{\frac{3}{3}}$ & th & 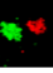 & * & 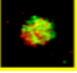 & $\frac{\widehat{\bar{c}}}{\sqrt[3]{3}}$ & $\%$ & 8 & t & 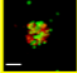 \\
\hline
\end{tabular}

Fig. 5 Identification of Ig1 specificity-determining residues.

(a) Ig1.30 domain structural modeling. Structural modeling shows that Ig1.30 domain might interact in an antiparallel fashion. Left: The residues represented in licorices (light orange) have 
been shown a complementary electrostatic potential surface pattern on the ABDE face: positive in one end, and negative in the other end. Right: Slices of the Ig1.30-Ig1.30 interface between strand A subunits. Potential neighboring interact residues (K5 and D12) were shown in licorices (light orange).

(b) The single disrupting and double complementary mutation of these candidate residues were assayed for cell aggregation. The double mutation partially rescued the reduced cell aggregates by single point mutations, supporting the antiparallel binding fashion.

(c) Residue swapping between sDscam $\alpha 20 \& \alpha 30 \& \alpha 36$ to assess specificity-determining residues. Left: Ig1 docking model and sequence alignments of shuffled regions are shown on the panel i. Four candidate specificity-determining residues (light orange) were located on adjacent B strands. Middle: Panel ii shows schematic representation of residue swapping mutants used in the experiments, along with a summary of results from binding specificity. Right: Panel iii shows the binding specificity of isoforms containing wild-type and swapped residue 22. Swapping of residue S1, S15, and V21 in Ig1.30 to Ig1.36 did not swap transbinding specificity (data not shown), while residue 22 swapping between $\operatorname{Ig} 1.30$ and $\operatorname{Ig} 1.36$ swapped binding specificity. See also Supplementary Fig. 5a.

(d) Residue swapping of variable $\operatorname{Ig} 1$ between sDscama21 and $\alpha 37$. Left panel (i) shows Ig1 docking model and sequence alignments of shuffled regions. Three candidate specificitydetermining residues (light orange) were located on adjacent A and D strands. Right panel (ii) shows the schematic diagrams of residue swapping mutants used in the experiments, along with observed binding specificity. Lower panel (iii) shows cell aggregation assays of isoforms containing wild-type and residue-swapped Ig1 domains. Swapping of either one of three residues between in Ig1.21 to Ig1.37 did not swap binding specificity, and swapping of two of three residues between in Ig1.21 to Ig1.37 partially swapped binding specificity, and swapping of all three residues between in Ig1.21 to Ig1.37 fully swapped binding specificity. See also Supplementary Fig. 5g. Scale bar, $100 \mu \mathrm{m}$. 

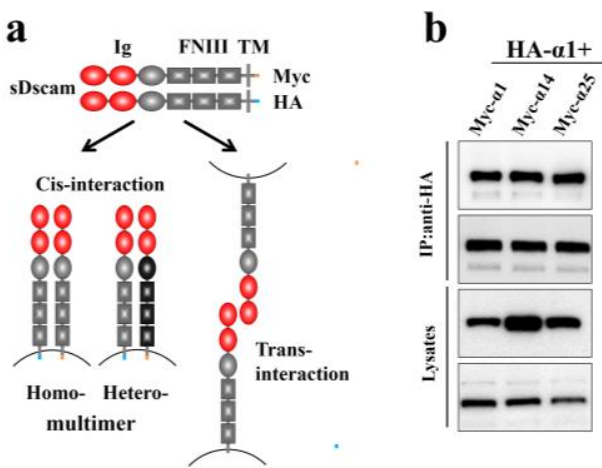

d

High-order multimerization assay
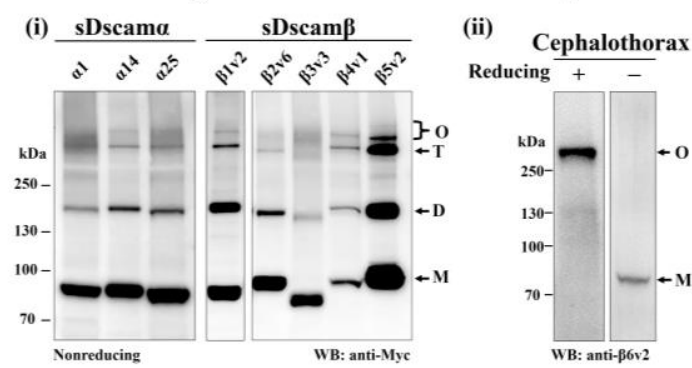

f

Multimerization of N-terminal truncations

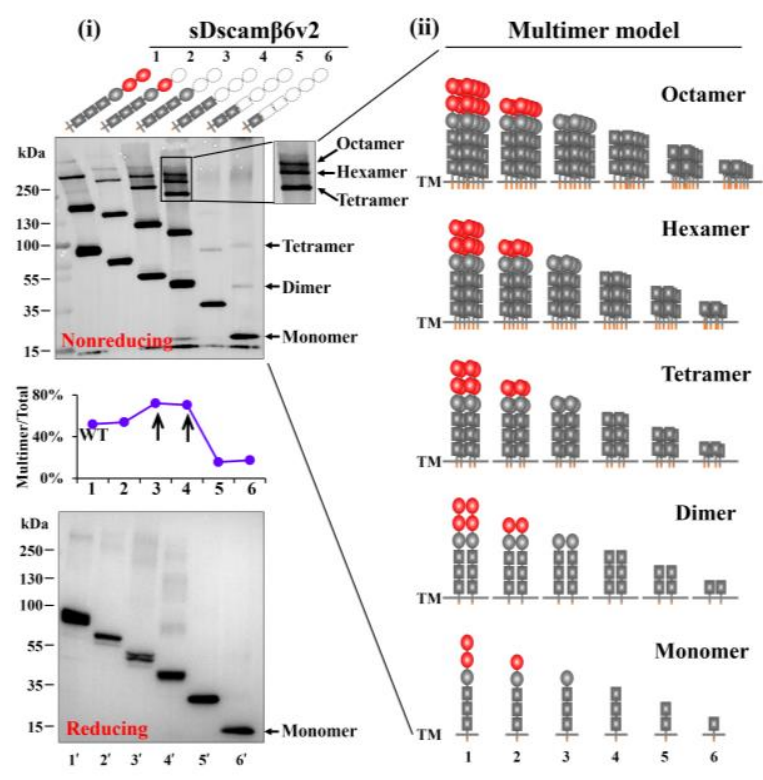

e Multimerization in the absence of trans interaction
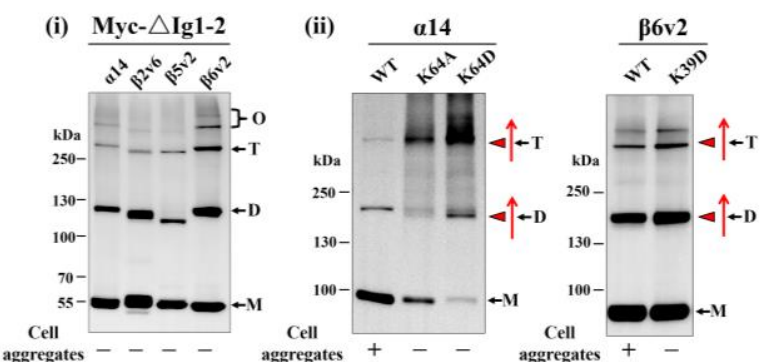

g FNIII1-3 domain co-IP and multimerization assay
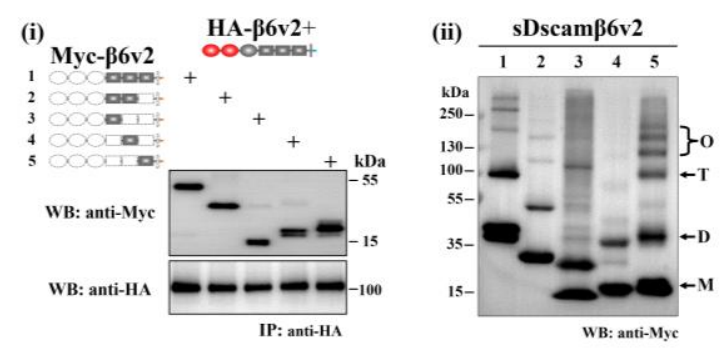

h

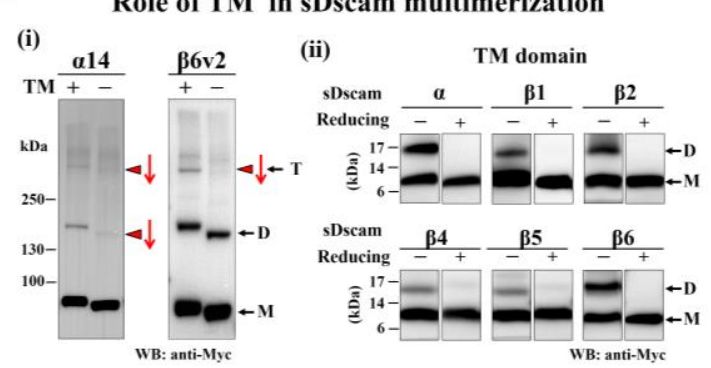

Fig. 6 sDscams form high order cis-multimers mediated by FNIII1-3 and TM domains.

(a) Schematic of cis- and trans- interaction of sDscam. sDscam monomers interacted in a parallel fashion to form a homomultimer or heteromultimer complex, while trans-multimers are formed between two opposing cells in an antiparallel fashion.

(b) All sDscam $\alpha$ and sDscam $\beta$ isoforms tested interacted strongly with each other in co-IP experiments. Lysates from Sf9 cells cotransfected with sDscam $\alpha 1$, sDscam $\beta 4 \mathrm{v} 1$ and 
sDscam $\beta 6 \mathrm{v} 2$ bearing a C-terminal HA-tag (HA- $\alpha 1$, HA- $\beta 4 \mathrm{v} 1$ and HA- $\beta 6 \mathrm{v} 2)$ and different Myctagged sDscam isoforms were immunoprecipitated using anti-HA antibody and probed with anti-Myc or anti-HA antibodies. See also Supplementary Fig.6a.

(c) sDscam $\beta 6 \mathrm{v} 2$ expressed in $\mathrm{Sf} 9$ cells formed cis-multimers. Lysates from $\mathrm{Sf} 9$ cells expressing sDscam $\beta 6 \mathrm{v} 2$ were run on SDS-PAGE in the presence of nonreducing or reducing agents, and analyzed by western blot with Myc antibody (lane 1-2), and with sDscam $\beta 6 \mathrm{v} 2$ antibody (lane $3-4)$.

(d) Multimerization assay of sDscam expressed in Sf9 cells (panel i) and from the scorpion cephalothorax (panel ii). Lysates from the scorpion cephalothorax were resolved on a SDS/PAGE gel under the nonreducing or reducing conditions, and analyzed by western blot with sDscam $\beta 6$ v2 antibody (panel ii).

(e) sDscams formed high order cis-multimers in the absence of trans interaction. (i) Proteins lacking Ig1-2, which have ablated homophilic trans-interactions, was able to form robust multimers. (ii) Single residue mutations (e.g., $\alpha 14$ K64A, $\alpha 14$ K64D and $\beta 6 v 2 K 39 D$ ), which ablated homophilic cell aggregation, caused increased multimerization.

(f) A series of N-terminal truncations of the extracellular domain of sDscam $\beta 6 \mathrm{v} 2$ fused with Myc-tag were examined for multimerization assay. Nonreducing (upper panel i) or reducing (lower panel i) SDS/PAGE gels were analyzed, with graph of the ratio change of multimer/total below nonreducing gel. Schematic architecture of $c i s$-multimer was depicted on the right (panel ii). See also Supplementary Fig.6e.

(g) Co-IP and multimerization assay of FNIII1-3s. sDscam $\beta 6$ v2 interacted strongly with each truncated protein expressing individual or combined domain of FNIII1-3s (panel i), and each truncated protein could form strong cis-multimers (panel ii).

(h) Transmembrane domain promotes the formation of sDscam cis-multimers. TM domain deletion strikingly reduced sDscam multimerization (Downward red arrow, panel i). The TM peptides expressed from various sDscams could dimerize strongly (panel ii). See also Supplementary Fig.6g. 
a

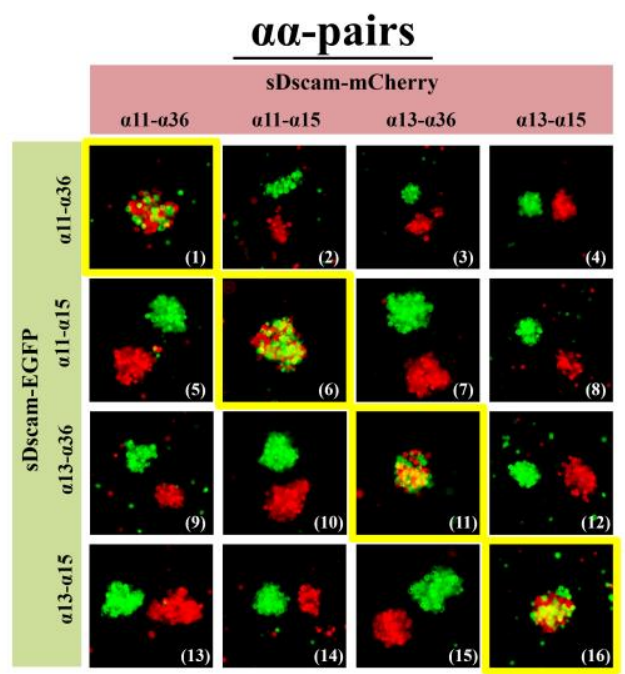

b

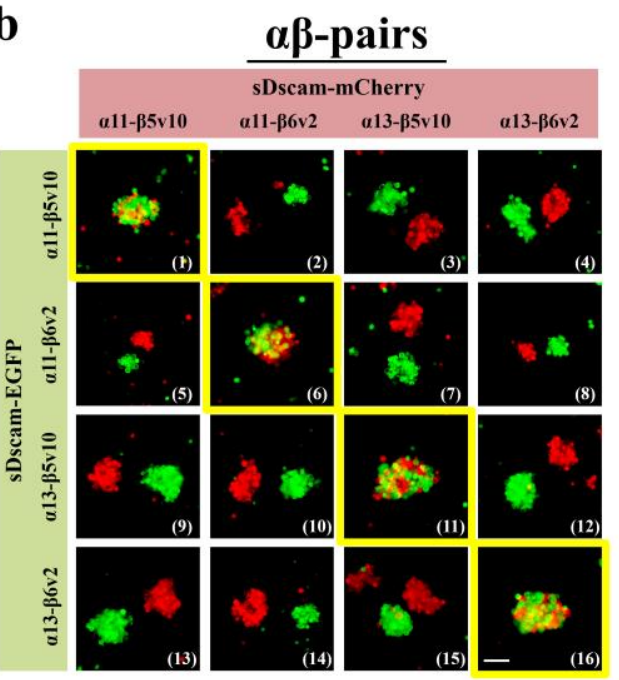

c

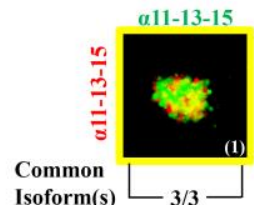

d

Cis-multimeric sDscam recognition unit

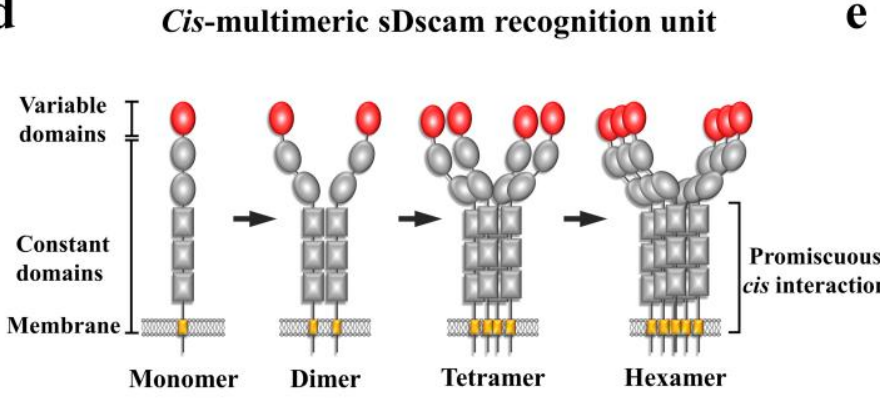

f

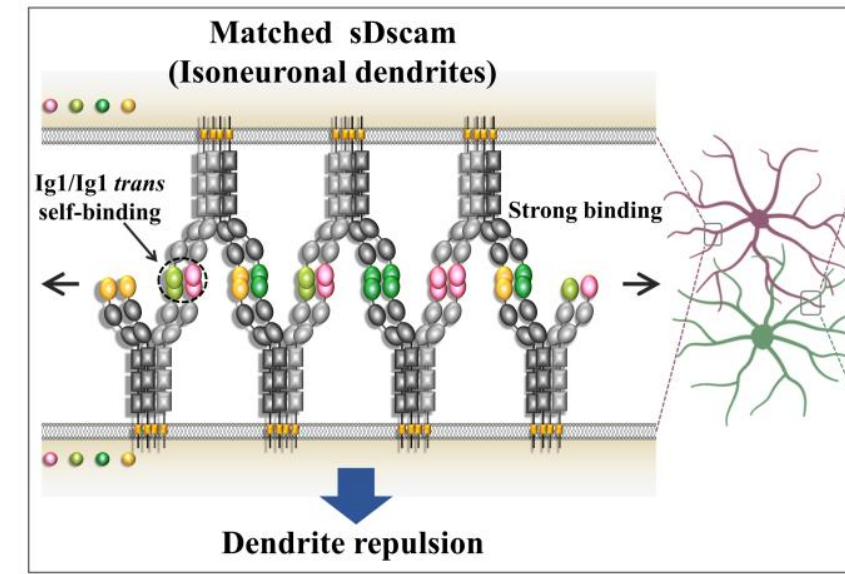

$\mathbf{e}$
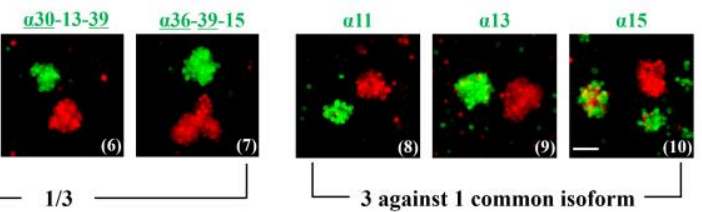

Fig. 7 The model for sDscam-mediated cell-cell recognition.

(a, b) Combinatorial coexpression of multiple sDscam isoforms generates unique cell surface 
identities. Cells coexpressing an identical or a distinct set of sDscam $\alpha(\mathbf{a})$ and $\mathrm{sDscam} \beta 5-\beta 6$ isoforms (b) were assayed for coaggregation. See also Supplementary Fig.7a.

(c) Cells coexpressing three distinct mCherry-tagged sDscam isoforms were assayed for interaction with cells expressing an identical or a distinct set of GFP-tagged sDscam isoforms. The nonmatching isoforms between two cell populations are underlined. See also Supplementary Fig.7a. Scale bar used in $(\mathrm{a}-\mathrm{c}), 100 \mu \mathrm{m}$.

(d) Model of cis-multimeric sDscam recognition units. The cis interface is located on longrange region from FNIII1 to TM domains. Since all sDscam assembly sizes were the fold of dimer, we suggest that dimer might act as basic recognition units for sDscam, and then clustered into tetramer and higher orderly oligomeric complexes.

(e) The model for sDscam-mediated cell-cell recognition. In this model, each sDscam cistetramers could interact multiple cis-tetramers on apposed cell surfaces via independent trans Ig1/Ig1 self binding, thereby forming a connected latticed assembly of proteins between cells.

(f) The model of sDscam-mediated neuronal self-recognition and self-non-self discrimination. Due to identical sDscam isoforms in two neurites of the same neuron, the trans-dimerization of cis-multimers could lead to a dense and connected lattice assembly between two apposing cell surfaces, thus triggering strong homophilic interactions and then inducing neurite repulsion. In contrast, mismatched Ig1s between two neighbouring neurons lead to a scattered or sparse connected lattice assembly between apposing cell surfaces, triggering weak homophilic interactions. Thus, the resulting downstream signaling is below the threshold level and fails to initiate neurite repulsion. 


\section{Convergent molecular strategies for self-avoidance}

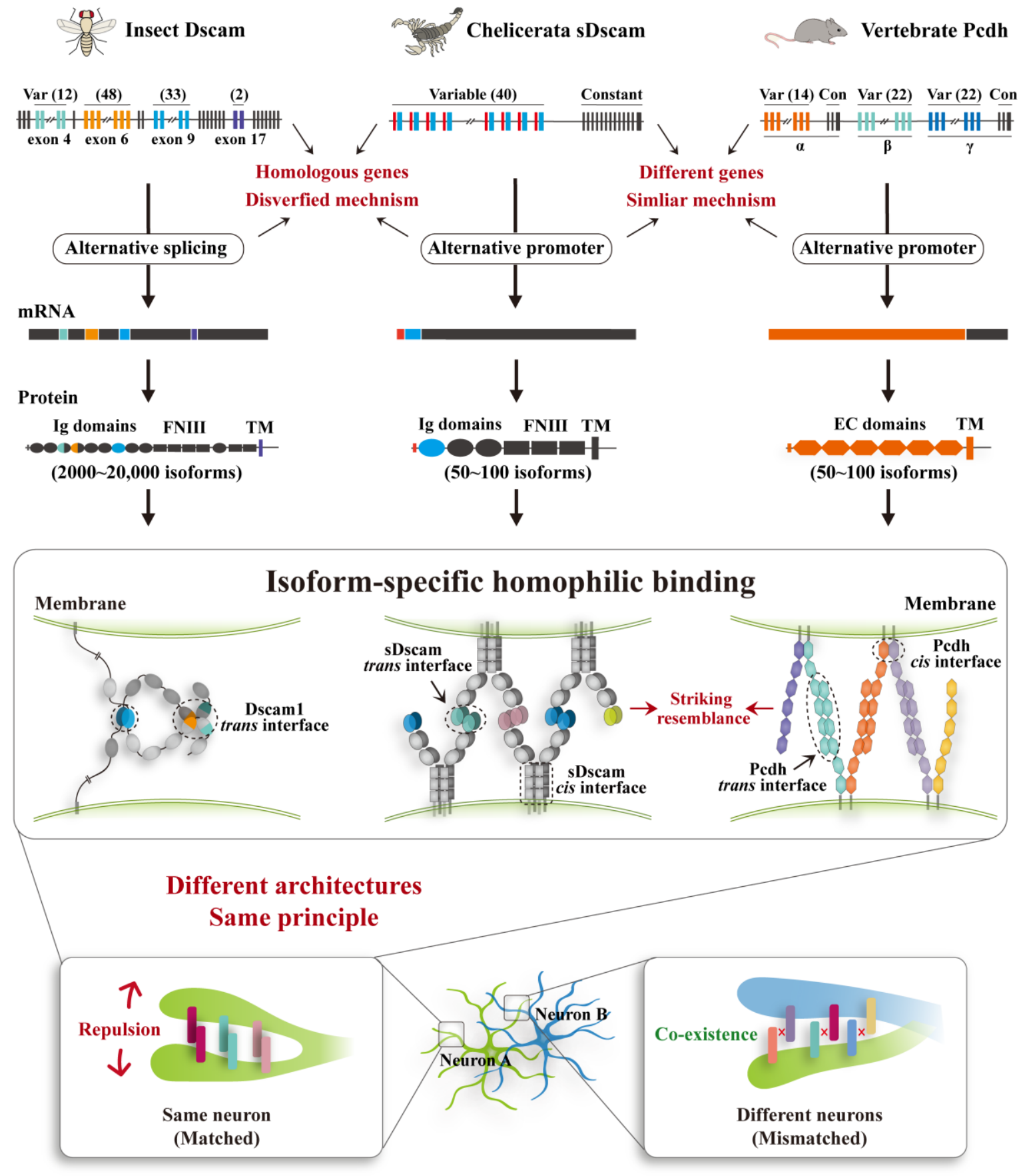

Self-recognition: Distinguishing self from non-self

Fig. 8 Chelicerata sDscams show more parallels with vertebrate Pcdhs than Drosophila

\section{Dscam1.}


Chelicerata sDscams show more parallels with vertebrate Pcdhs than Drosophila Dscam1. Drosophila Dscam1 generates ten thousands of protein isoforms through alterntive splicing 9 . Chelicerata sDscam genes employ alternative promoter to generate extensive isoforms as vertebrate Pcdhs ${ }^{17,33}$. Three of them encode a large number of single-transmembrane protein isoforms, and the encoded proteins engage in isoform-specific homophilic binding. This suggests that different phyla seem to have used different molecules to mediate analogous principle for self-recognition and self-non-self discrimination during neuronal arborization. However, in contrast to fly Dscam1 isoforms which was shown to act as cis-monomer, sDscam and Pcdh proteins act as cis-multimeric recognition units to expand adhesive interfaces. 